\title{
Validation of International Atomic Energy Agency Equipment Performance Requirements
}

February 2004

\author{
Prepared by \\ Peter J. Chiaro, Jr. \\ Fred R. Gibson \\ Kathy L. Johnson
}




\title{
DOCUMENT AVAILABILITY
}

Reports produced after January 1, 1996, are generally available free via the U.S. Department of Energy (DOE) Information Bridge:

Web site: http://www.osti.gov/bridge

Reports produced before January 1, 1996, may be purchased by members of the public from the following source:

\author{
National Technical Information Service \\ 5285 Port Royal Road \\ Springfield, VA 22161 \\ Telephone: 703-605-6000 (1-800-553-6847) \\ TDD: 703-487-4639 \\ Fax: 703-605-6900 \\ E-mail: info@ntis.fedworld.gov \\ Web site: http://www.ntis.gov/support/ordernowabout.htm
}

Reports are available to DOE employees, DOE contractors, Energy Technology Data Exchange (ETDE) representatives, and International Nuclear Information System (INIS) representatives from the following source:

Office of Scientific and Technical Information

P.O. Box 62

Oak Ridge, TN 37831

Telephone: 865-576-8401

Fax: 865-576-5728

E-mail: reports@adonis.osti.gov

Web site: http://www.osti.gov/contact.html

This report was prepared as an account of work sponsored by an agency of the United States Government. Neither the United States government nor any agency thereof, nor any of their employees, makes any warranty, express or implied, or assumes any legal liability or responsibility for the accuracy, completeness, or usefulness of any information, apparatus, product, or process disclosed, or represents that its use would not infringe privately owned rights. Reference herein to any specific commercial product, process, or service by trade name, trademark, manufacturer, or otherwise, does not necessarily constitute or imply its endorsement, recommendation, or favoring by the United States Government or any agency thereof. The views and opinions of authors expressed herein do not necessarily state or reflect those of the United States Government or any agency thereof.

\section{COPYRIGHT NOTICE}

The submitted manuscript has been authored by a contractor of the U.S. Government under contract DE-AC05-00OR22725. Accordingly, the U.S. Government retains a paid-up, nonexclusive, irrevocable, worldwide license to publish or reproduce the published form of this contribution, prepare derivative works, distribute copies to the public, and perform publicly and display publicly, or allow others to do so, for U.S. Government purposes. 
ORNL/TM-2004/26

\section{Validation of International Atomic Energy Agency Equipment Performance Requirements}

Peter J. Chiaro, Jr.

Fred R. Gibson

Kathy L. Johnson

Date Published: February 2004

Prepared by

OAK RIDGE NATIONAL LABORATORY

P.O. Box 2008

Oak Ridge, Tennessee 37831-6285

managed by

UT-Battelle, LLC

for the

U.S. DEPARTMENT OF ENERGY

under contract DE-AC05-00OR22725 


\section{CONTENTS}

Page

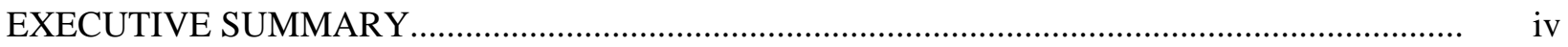

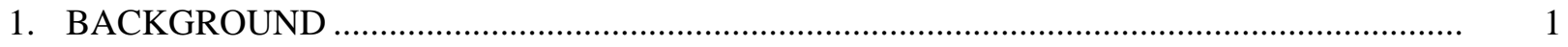

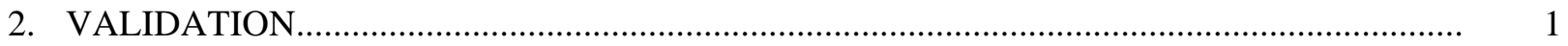

3. PERFORMANCE SPECIFICATION RECOMMENDED CHANGES ...................................... 1

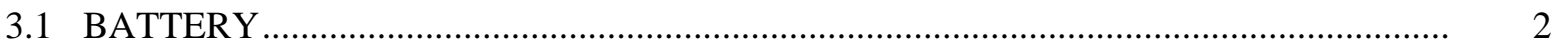

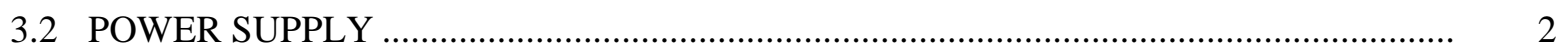

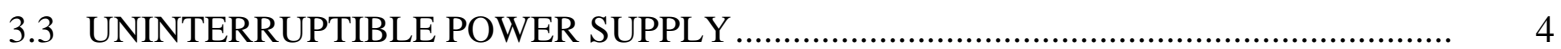

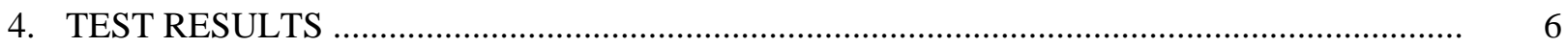

APPENDIX A. IAEA PERFORMANCE SPECIFICATION BATTERY ........................................ 8

APPENDIX B. IAEA PERFORMANCE SPECIFICATION POWER SUPPLY ............................... 9

APPENDIX C. IAEA PERFORMANCE SPECIFICAITON UNINTERRUPTIBLE

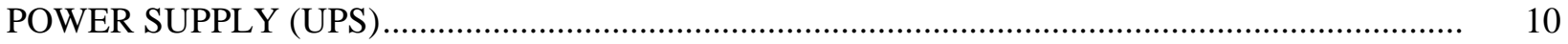

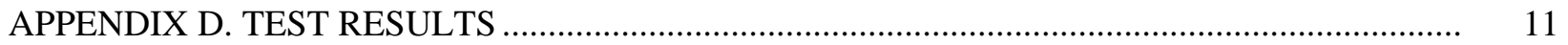

APPENDIX E. EQUIPMENT AND INSTRUMENTS USED IN TESTING................................... 12 


\section{EXECUTIVE SUMMARY}

Performance requirements and testing protocols are needed to ensure that equipment used by the International Atomic Energy Agency (IAEA) is reliable. Oak Ridge National Laboratory (ORNL), through the U. S. Support Program, tested equipment to validate performance requirement protocols used by the IAEA for the subject equipment categories. Performance protocol validation tests were performed in the Environmental Effects Laboratory in the categories for battery, DC power supply, and uninterruptible power supply (UPS). Specific test results for each piece of equipment used in the validation process are included in this report.

The results of these tests validate the performance requirements developed by ORNL for use by the IAEA. 


\section{BACKGROUND}

The International Atomic Energy Agency (IAEA) is an organization whose mission is to ensure that nuclear science and technology is applied to international peace and security efforts. Performance requirements and testing protocols are needed to ensure that equipment used by the IAEA is reliable. Oak Ridge National Laboratory (ORNL), through the U. S. Support Program, was asked to test equipment in three categories to validate the performance requirement protocols used by the IAEA for the subject equipment categories. Performance protocol validation tests were performed in the Environmental Effects Laboratory in the categories for battery, DC power supply, and uninterruptible power supply (UPS).

\section{VALIDATION}

One of the greatest challenges of performance requirements validation is separating the equipment test results from the validation process. It is imperative that the equipment is not considered for its success or failure but that the process for introducing that equipment into use as an international safeguard device is appropriate, consistent, and applicable to a wide variety of devices. In order to ensure these goals are met, each device was subjected to the specific requirements for use as stated in the IAEA performance specification for that equipment. The testing process for each protocol validation was a step-by-step process dictated by the protocol. Tests were setup based on International Electrotechnical Commission (IEC) standards and other consensus standards referenced by the ORNL-developed IAEA performance specifications.

The equipment used in this protocol validation were:

- Sonnenschein A500 battery,

- Sonnenschein A200 battery,

- Panasonic LC-R123R4 battery,

- Lambda 18V power supply,

- APC SU2200XL UPS,

- Trace SW3048J UPS, and

- $\quad$ Trace DR1512E UPS.

Each device was chosen for testing and provided by the IAEA. The devices were subjected to environmental tests (temperature, humidity and condensation), vibration, radiated and conducted radio frequency (RF) emissions, electrical fast transient, surge, and shock tests. The environmental tests included power supply dip/interruption evaluations, where appropriate.

\section{PERFORMANCE SPECIFICATION RECOMMENDED CHANGES}

Each test was carefully setup within the bounds of the protocol. Equipment performance was evaluated based on the protocol requirements for use. As a result of the testing, it was determined that changes 
should be made to the performance specifications. Protocol changes improved readability and test flow. In addition the changes ensure that the tests are consistent with the expected conditions of use.

The changes to the individual sections of each protocol are shown with the original text red-lined followed by the revised text in italics. The complete red-lined versions are attached as Appendix A, B, and C, respectively.

\subsection{BATTERY}

IAEA Safeguards systems may incorporate the use of batteries to either provide transfer power for a UPS device or as direct power for system operation. Typically, a $12 \mathrm{~V}$ battery would be used. Based on the test results, the following changes have been made to section $\underline{4}$. Testing Protocol.

4.b.i Connect a fully charged battery to the test load (the test load shall be equivalent to $75 \%$ of the stated maximum load for the selected battery type the expected load for battery application.)

4.b.ii With the load stable, measure the output voltage and current of the battery. Chart the results voltage and current as a function of time for $z 4$ hours, of an appropriate time based on the manufacturer-stated battery capacity.

4.b.iii Record when the battery's capacity becomes less than the IAEA required battery minimum recommended capacity (needs to be determined approximately when load voltage $=9 \mathrm{~V}$ ).

4.c.iv After the test is complete, disconnect the charger, attach the test load, and perform a function test.

\section{4.d Vibration}

The vibration test is based on conditions stated in IEC 60721-3-3, Class 3M4 and 60068-2-6 Fc:

Vibration sinusoidal. Each battery shall be functionally tested prior to and after each exposure each exposure, during exposure, and after each axis is finished. Monitor battery no-load voltage during exposure.

\section{4.d.i.3 Axes $=31$}

\section{4.d.i.4 Sweep Cycles $=10$ (approximately 2 hours per axis)}

\section{4.e $\underline{\text { Shock }}$}

The shock (bump) test is based on conditions stated in IEC 60721-3-3, Class 3M4 and 60068-2-29

Eb: Bump. Each battery shall be functionally tested prior to and after each exposure, during exposure, and after each axis is finished. Monitor battery no-load voltage during exposure.

4.e.i.3 Number of shocks/bumps $=100$ in each direction

4.e.i.4 Direction $=31$

\subsection{POWER SUPPLY}

Direct Current (DC) power supplies may be used to provide stable power to various IAEA Safeguards system components, which could include the control computer. Power supplies may be operated in different environments but would not include any unexpected ionizing radiation fields. Power supplies 
are tested at $100 \%$ of the stated maximum load for the selected device. Based on the test results, changes

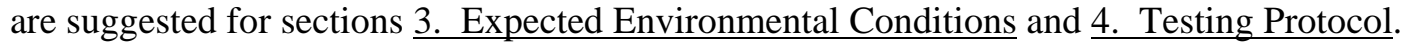

\section{3.l Electrical fast transient (Burst) (IEC references 61000-4-4)}

The power supply shall function normally when exposed to the following electrical fast transients on the mains input (severity level 4 - severe industrial environment):

\begin{tabular}{|c|c|}
\hline $\begin{array}{c}\text { Voltage Peak } \\
\text { KV }\end{array}$ & $\begin{array}{c}\text { Repetition Rate } \\
\mathbf{k H z}\end{array}$ \\
\hline 42 & 100 \\
\hline
\end{tabular}

3.m Surge (IEC references 61000-4-5)

The power supply shall function normally when exposed to the following transients on the mains input: $1.2 / 50 \mu \mathrm{s}-8 / 20 \mu$ s combination waveform at an intensity of $42 \mathrm{kV}$

4.b.ii Slowly reduce the input voltage in $5 \%$ increments until reaching the 15\% voltage and Record the following information:

4.b.ii.1 Output $D C$ voltage level and output ripple voltage (rms value)

4.b.iii Slowly reduce the input voltage until reaching -15\% voltage and repeat step 4.b.ii.

4.b.v Slowly increase the input voltage in $5 \%$ increments until reaching the $+15 \%$ voltage and record the following information: repeat step 4.b.ii.

1. Output voltage level

Z. Line perturbations

Line perturbations shall be within the following limits (Class A from NF EN 55022 standard):

\begin{tabular}{|l|ll|}
\hline $\begin{array}{l}\text { Frequency } \\
\text { fange } \\
(\mathrm{MHz})\end{array}$ & Limits & $\mathrm{dB}(\mu \mathrm{V})$ \\
\cline { 2 - 3 } & $\mathrm{Q}$-Peak & Average \\
\hline 0.15 to 0.50 & 79 & 66 \\
\hline 0.5 to 30 & 73 & 60 \\
\hline
\end{tabular}

4.b.vi Repeat step 4.b.iv.

4.b.vii Connect the power supply to a test load (the test load shall be equivalent to $50 \%$ of the stated maximum load for the selected unit.) Repeat step 4.b.ii.

4.b.viii Disconnect the power supply from test load (no load). Repeat step 4.b.ii. Reconnect the power supply to $100 \%$ load.

4.d.iv These conditions shall be maintained for 96 hours, during which the power supply is tested per steps 4.b.ii through 4.b.vi viii at one 12-hour intervals.

4.d.vi Once the power supply reaches thermal equilibrium, its function shall be tested per 4.b.ii through 4.b.ivix.

4.e.viii Prior to temperature change steps, the power supply's function shall be tested per steps 4.b.ii through 4.b. $\forall$ viii and results recorded. 
4.e.ix Once the second cycle is complete, reduce the temperature and RH level to the reference conditions and re-test the eharger power supply per steps 4.b.ii through 4.b. vi $i x$.

\section{4.f $\underline{\text { Vibration }}$}

The vibration test is based on conditions stated in IEC 60721-3-3, Class 3M4 and 60068-2-6 Fc: Vibration sinusoidal. The power supply shall be functionally tested per step 4.b prior to each exposure,-during exposure, and the output voltage monitored during exposure. After each axis is finished the power supply shall be functionally tested per step 4.b.

\section{4.g Shock}

The shock (bump) test is based on conditions stated in IEC 60721-3-3, Class 3M4 and 60068-2-29 Eb: Bump. The power supply shall be functionally tested per step 4.b prior to each exposure and the output voltage monitored during exposure. After each axis is finished the power supply shall be functionally tested per step 4.b. The power supply shall be functionally tested per step -4.b prior to each exposure, during exposure, and after each axis is finished.

\section{4.h Radiated emissions}

RF emissions shall not exceed the levels stated below. Tests shall be performed using a SAS antenna or equivalent device and a spectrum analyzer. Emissions measurements shall be made with the antenna placed one meter from the center of the power supply. Measurements shall be made with the power supply functioning with full line voltage and maximum load.

4.i.i Prior to testing, the power supply shall be functioning normally and operated tested based on 4.b.ii through 4.b.vi $i x$.

4.i.ii During exposures, the output voltage and voltage waveform shall be monitored according to section-4.b.

4.j Conducted disturbances induced by radio frequencies

The fields shown below will be applied to the input lines through a coupling/decoupling network. During exposure, the power supply will output voltage and voltage waveform shall be functionally tested using guidance found in 4 .b.i through vi monitored.

\section{4.k Radiated radio frequency fields}

The power supply shall be exposed to the fields shown below. During exposure, the power supply will output voltage and voltage waveform shall be functionally tested using guidance found in 4 .b.i through vi monitored.

4.m.iii Each pulse shall consist of a combination wave $(1.2 / 50 \mu \mathrm{s}-8 / 20 \mu \mathrm{s})$ at an intensity of $42 \mathrm{kV}$

\subsection{UNINTERRUPTIBLE POWER SUPPLY}

Uninterruptible power supply (UPS) devices are incorporated in most IAEA safeguards systems to maintain system power and permit some level of functionality in case of power failure. These devices can be expected to function in various environments but unexpected ionizing radiation fields would not be included. Based on the test results, the following suggestions for changes to the performance specifications for UPS devices are made in sections 3. Expected environmental conditions and 4. Testing Protocol. 
3.I The UPS shall function normally when exposed to the following electrical fast transients on the mains input (severity level 4 - severe industrial environment):

\begin{tabular}{|c|c|}
\hline $\begin{array}{c}\text { Voltage Peak } \\
\text { KV }\end{array}$ & $\begin{array}{c}\text { Repetition Rate } \\
\mathbf{k H z}\end{array}$ \\
\hline 42 & 100 \\
\hline
\end{tabular}

3.m The UPS shall function normally when exposed to the following transients on the mains input: $1.2 / 50 \mu \mathrm{s}-8 / 20 \mu \mathrm{s}$ combination waveform at an intensity of $42 \mathrm{kV}$.

4.d.iv These conditions shall be maintained for 96 hours, during which the UPS is functionally tested (4.b.ii through vi) at one 12-hour intervals.

4.3.ix Once the second cycle is complete, reduce the temperature and $\mathrm{RH}$ level to the reference conditions and re-test the UPS (4.b.ii through vi).

\section{4.f $\underline{\text { Vibration }}$}

The vibration test is based on conditions stated in IEC 60721-3-3, Class 3M4 and 60068-2-6 Fc: Vibration sinusoidal. The UPS shall be functionally tested prior to each exposure,during expesure, and after each axis is finished. The output voltage shall be monitored during exposure.

\section{4.g Shock}

The shock (bump) test is based on conditions stated in IEC 60721-3-3, Class 3M4 and 60068-2-29

Eb: Bump. The UPS shall be functionally tested prior to each exposure,-during exposure, and after each axis is finished. The output voltage shall be monitored during exposure.

\section{4.h Radiated emissions}

RF emissions shall not exceed the levels stated below. Tests shall be performed using a SAS antenna or equivalent device and a spectrum analyzer. Emissions measurements shall be made with the antenna placed one meter from the center of the UPS. Measurements shall be made with the UPS monitoring line power (pass-through mode) and with the UPS functioning (inverter mode).

4.i.ii During exposures, the output line voltage and voltage waveform shall be monitored according to section 4.b.

4.j Conducted disturbances induced by radio frequencies

The fields shown below will be applied to the mains through a coupling/decoupling network. During exposure, the UPS will be functionally tested using guidance found in 4 .b.ii through vi output voltage and voltage waveform shall be monitored. Testing shall be done in both the pass-through mode and the inverter mode.

4.k Radiated radio frequency fields

The UPS shall be exposed to the fields shown below. During exposure, the UPS will be functionally tested using guidance found in 4.b.ii through vi output voltage and voltage waveform shall be monitored. Testing shall be done in both the pass-through mode and the inverter mode.

4.m.iii Each pulse shall consist of a combination wave $(1.2 / 50 \mu \mathrm{s}-8 / 20 \mu \mathrm{s})$ at an intensity of $42 \mathrm{kV}$. 


\section{TEST RESULTS}

A brief summary of the test results from each device follows. All batteries were subjected to temperature testing. One battery was also tested for vibration and shock. Specific test results are shown in Appendix $\mathrm{D}$ attached to this report. A brief description of the test equipment used for conducting all tests described in this report is attached as Appendix E.

1. Sonnenschein A500 battery. No susceptibilities were observed during the temperature test. No functional problems were observed during testing.

2. Sonnenschein A200 battery. No susceptibilities were observed during the temperature test. No functional problems were observed during testing.

3. Panasonic LC-R123R4 battery. No susceptibilities were observed during the temperature test. No functional problems were observed during testing.

4. Lambda $18 \mathrm{~V}$ power supply. The tests performed and summary of the observations for this device are:

- $\quad$ Temperature - No susceptibilities were observed. No functional problems were observed during testing.

- Humidity - No susceptibilities were observed. No functional problems were observed during testing.

- Condensation - No susceptibilities were observed. No functional problems were observed during testing.

- Vibration - No susceptibilities were observed. No functional problems were observed during testing.

- Shock - No susceptibilities were observed. No functional problems were observed during testing.

- RF Emissions - No significant emissions were observed during testing.

- Electrical Discharge - A “burst” waveform (0.7ms duration, 4.6vp-p contact, 8.4vp-p air) was observed on the output of the supply at full load conditions. The power supply continued functioning during the test and afterwards.

- RF Field - No susceptibilities were observed. No functional problems were observed during testing.

- Conducted RF - No susceptibilities were observed. No functional problems were observed during testing.

- Electrical burst - No susceptibilities were observed. No functional problems were observed during testing.

- Electrical surge - No susceptibilities were observed. No functional problems were observed during testing.

5. APC SU2200XL UPS - The tests performed and a summary of the results are:

- Temperature - No susceptibilities were observed. No functional problems were observed during testing.

- Electrical Discharge - No susceptibilities were observed. No functional problems were observed during testing. 
6. Trace SW3048J UPS - The tests performed and a summary of the results are:

- Condensation - No susceptibilities were observed. No functional problems were observed during testing.

- Electrical Discharge - No susceptibilities were observed. No functional problems were observed during testing.

- Electrical burst - No susceptibilities were observed. No functional problems were observed during testing.

- Electrical surge - Susceptibilities were observed during this test. When subjected to a $4 \mathrm{kV}$ combination wave, the UPS failed with the second pulse. It continuously switched back and forth between mains to inverter. No further testing could be performed on this device.

7. Trace DR1512E - The tests performed and a summary of the results are:

- Temperature - No susceptibilities were observed. No functional problems were observed during testing.

- Humidity - No susceptibilities were observed. No functional problems were observed during testing.

- Vibration - No susceptibilities were observed. No functional problems were observed during testing.

- Shock - No susceptibilities were observed. No functional problems were observed during testing.

- RF Emission - No susceptibilities were observed. No functional problems were observed during testing.

- Electrical Discharge - Susceptibilities were observed during this test. An air discharge (8kv) was applied near the "battery low/high" light which shutdown the UPS while under load. The on/off button was pressed and the UPS restarted.

- $\quad$ RF Field - Susceptibilities were observed between 107 to $122 \mathrm{MHz}$ and 149 to $151 \mathrm{MHz}$ during this test.

- Conducted RF - Susceptibilities were observed between 60 to $80 \mathrm{MHz}$ during this test. 
APPENDIX A

IAEA Performance Specification

Battery

6 December 2002 


\section{IAEA Performance Specification Battery \\ 6 December 2002}

\section{Background}

IAEA Safeguards systems may incorporate batteries that can provide transfer power for the UPS device, or direct power for system operation. Typically, the batteries used provide a nominal 12 VDC.

The load for testing purposes shall be $75 \%$ of the stated maximum capacity of the selected battery.

\section{Requirements for Use}

1. Functional

a. Recharge cycles - 700

b. Expected operational lifetime - 5 years

c. Expected shelf life No specific requirement, but self-discharge should be as low as practical.

d. Procurement age When procured, batteries must be less than 6-months old.

e. Output power

i. Voltage Typically 12 volts.

ii. Capacity

Selection made based on the functional requirements of the associated equipment. No requirement is defined.

b. Data communication

No requirement.

c. Radiated emissions

Not applicable.

2. Mechanical design

a. Size

No requirement defined. Determination will be made based on application.

b. Weight

No requirement defined. Determination will be made based on application.

c. Internal

Acid gel

d. Maintenance

Batteries shall be maintenance free.

e. Venting

Batteries must contain an appropriate amount of automatic overpressure valves.

f. Connectors

No requirement defined. Selection based on specific application.

3. Expected environmental conditions

a. Ambient temperature

$+5{ }^{\circ} \mathrm{C}$ to $+45^{\circ} \mathrm{C}$ (based on $60721-4-3$, class $3 \mathrm{~K} 4$ )

b. Relative humidity

No defined requirement. 
c. Condensation

No defined requirement.

d. Atmospheric pressure

No defined requirement.

e. Dust and splash water

The IAEA cabinet is required to meet IP56 (Dust Proof and Spray Proof, respectively); therefore no requirement is stated for individual components. (EN 60529/IEC 529).

f. Vibration

The battery(s) is expected to function normally when exposed to vibration conditions of up to $10 \mathrm{~m} / \mathrm{s}^{2}$ over a frequency range from $5 \mathrm{~Hz}$ to $150 \mathrm{~Hz}$.

g. Shock

The battery(s) is expected to function normally when exposed to shock conditions of up to $150 \mathrm{~m} / \mathrm{s}^{2}$.

h. Electrostatic discharge

No defined requirement.

i. Conducted disturbances induced by radio frequencies

No defined requirement.

j. $\quad$ Radiated radio frequency fields

No defined requirement.

k. Voltage dips and short interruptions

No defined requirement.

1. Electrical fast transient (Burst) (IEC references 61000-4-4)

No defined requirement.

m. Surge (IEC references 61000-4-5)

No defined requirement.

4. Testing protocol

a. Nominal environmental conditions

i. $20^{\circ} \mathrm{C} \pm 2{ }^{\circ} \mathrm{C}, 40-75 \% \mathrm{RH}$

ii. Ambient electromagnetic (EM) fields less than those that can cause interference.

b. Function

i. Connect a fully charged battery to the test load (the test load shall be equivalent to $75 \%$ of the stated maximum load for the selected battery type the expected load for battery application).

ii. With the load stable, measure the output voltage and current of the battery. Chart the results-voltage and current as a function of time for $z 4$ hours, or an appropriate time based on the manufacturer-stated battery capacity.

iii. Record when the battery's capacity becomes less than the IAEA required battery minimum recommended capacity (needs to be determined approximately when load voltage $=9 \mathrm{~V}$ ).

iv. Remove the test load and recharge the battery per the manufacturer's recommendations.

v. Record the time required for the battery to reach maximum capacity, and the voltage and current during the charging process.

c. Temperature

The following temperature test is based on conditions stated in IEC 60721-4-3: Stationary use at weather protected locations - class 3K4, 60068-2-2 Bb/Bd: Dry heat, and 60068-2-1 Ab/Ad: Cold. 
i. Place each battery into an environmental chamber. Allow the chamber to stabilize at the reference conditions $\left(20^{\circ} \mathrm{C} \pm 2{ }^{\circ} \mathrm{C}, 40-75 \% \mathrm{RH}\right)$.

ii. Reduce the temperature to $+5{ }^{\circ} \mathrm{C}$ at a rate not exceeding $10^{\circ} \mathrm{C} / \mathrm{hr}$. The relative humidity level should not exceed the reference range during this time. The temperature shall be maintained at $+5{ }^{\circ} \mathrm{C}$ for at least 16 hours once the battery(s) reaches equilibrium with the ambient temperature.

iii. Perform a "Function" test per 4.b.i through v.

iv. After the test is complete, disconnect the charger, attach the test load, and perform a function test.

v. The temperature shall then be increased at a rate not exceeding $10^{\circ} \mathrm{C} / \mathrm{hr}$ to $+45^{\circ} \mathrm{C}$.

vi. Repeat steps 4.c.iii and 4.c.iv.

vii. After the high temperature exposure, return the temperature to the reference level and repeat steps 4.c.iii through 4.c.iv.

viii. Document all results and report any functional abnormalities to the

d. Vibration appropriate task leader.

The vibration test is based on conditions stated in IEC 60721-3-3, Class 3M4 and 60068-2-6 Fc: Vibration sinusoidal. Each battery shall be functionally tested prior to and after each exposureeach exposure, during exposure, and after each axis is finished. Monitor battery no-load voltage during exposure.

i. Vibration parameters are as follows:

1. Acceleration $=10 \mathrm{~m} / \mathrm{s}^{2}$

2. Frequency Range $=5 \mathrm{~Hz}$ to $150 \mathrm{~Hz}$

3. Axes $=31$

4. Sweep Cycles $=10$ (approximately 2 hours per axis)

ii. Document all results and report any functional abnormalities to the appropriate task leader.

e. Shock

The shock (bump) test is based on conditions stated in IEC 60721-3-3, Class 3M4 and 60068-2-29 Eb: Bump. Each battery shall be functionally tested prior to and after each exposure,during exposure, and after each axis is finished. Monitor battery no-load voltage during exposure.

i. Shock test parameters are as follows:

1. Peak Acceleration $=150 \mathrm{~m} / \mathrm{s}^{2}$

2. Duration $=6 \mathrm{~ms}$

3. Number of shocks/bumps $=100$ in each direction

4. Direction $=31$

ii. Document all results and report any functional abnormalities to the appropriate task leader. 


\section{APPENDIX B}

\section{IAEA Performance Specification}

Power Supply

6 December 2002 


\section{IAEA Performance Specification \\ Power Supply \\ 6 December 2002}

\section{Background}

Some IAEA Safeguards systems incorporate DC power supplies that are used to provide stable DC power to various components contained within the safeguards system. These components could include the control computer.

Power supplies may be used in different environments, which do not include any unexpected ionizing radiation fields.

The load for testing purposes shall be $100 \%$ of the stated maximum load for the selected power supply.

\section{Requirements for Use}

1. Functional

a. Input Power

i. Frequency

ii. Voltage

1. 50 or $60 \mathrm{~Hz}( \pm 5 \%)$

1. Nominal voltage is 100 to $230 \mathrm{VAC}( \pm 15 \%)$, or selectable between 100, 115, and 230 VAC.

2. Over voltage protection is required. Protection shall be activated if the input voltage exceeds the nominal value by more than $15 \%$.

iii. Current

1. The input current should be limited. Defined values are dependent on the specific application.

b. Output Power

i. DC output

1. Constant and stable to within $\pm 10 \%$ of the nominal voltage

2. Isolated from the chassis

3. Voltage and maximum current dependent on application (typically 12, 18, or 24 VDC)

ii. Over current protection

1. Automatic recovery required

iii. Over voltage protection shall be available

1. Manual reset

iv. Ripple noise

1. $<200 \mathrm{mV}$

v. Line regulation (affect from input line variations on output voltage)

1. $<100 \mathrm{mV}$

2. Addressed through voltage variation $( \pm 10 \%)$

vi. Load regulation (affect from 0 to full load on output voltage)

1. $<150 \mathrm{mV}$

vii. Line perturbations

During normal operations, line perturbations on the output power shall not exceed the following values: 


\begin{tabular}{|c|cc|}
\hline \multirow{2}{*}{$\begin{array}{c}\text { Frequency } \\
\text { range } \mathrm{MHz}\end{array}$} & Limits & $\mathrm{dB}(\mu \mathrm{V})$ \\
\cline { 2 - 3 } & $\mathrm{Q}-$ Peak & Average \\
\hline 0.15 to 0.50 & 79 & 66 \\
\hline 0.5 to 30 & 73 & 60 \\
\hline
\end{tabular}

c. Battery lifetime

i. Not applicable.

d. Electrical Safety Requirements

Power supplies shall meet appropriate electrical safety requirements. As a minimum, the unit shall meet appropriate ÖVE (Österreichischer Verein Der Elektrotechnik - Austrian Electrotechnical Association) requirements. The unit should also meet the requirements of the country of use.

e. Data Communication

i. No defined requirement.

f. Radiated emissions

Radiation emissions shall be less than the values shown below when measured at a distance of 1 meter (values are based on 10 meters and are from NF EN 55022 standard).

\begin{tabular}{|c|c|}
\hline Frequency range $\mathrm{MHz}$ & $\begin{array}{c}\text { Limits dB }(\mu \mathrm{V}) \\
\mathrm{Q}-\mathrm{Peak}\end{array}$ \\
\hline 30 to 230 & 40 \\
\hline 230 to 1000 & 47 \\
\hline
\end{tabular}

2. Mechanical Design

a. Size and weight

i. Determination based on specific application. No defined requirement.

b. Terminal connections

i. External connections are required

ii. Standard type contacts that permit easy cable removal without disassembling the power supply.

3. Expected Environmental Conditions

a. Ambient temperature

$+5{ }^{\circ} \mathrm{C}$ to $+45^{\circ} \mathrm{C}$ (based on $60721-4-3$, class $3 \mathrm{~K} 4$ )

b. Relative humidity

$+40{ }^{\circ} \mathrm{C}$ and $93 \%$

Need to meet tropical environment conditions (spent fuel bay) (3K4 shows +30

${ }^{\circ} \mathrm{C}$ and $93 \%$ for 96 hours). With required inspection, this test could cover the corrosion requirement.

c. Condensation

The power supply should be able to operate when exposed to a condensing atmosphere.

d. Atmospheric pressure

No defined requirement.

e. Dust and Splash Water

The IAEA cabinet is required to meet IP56 (dust proof and spray proof, respectively); therefore no requirement is stated for individual components. (EN 60529/IEC 529). 
f. Vibration

The power supply is expected to function normally when exposed to vibration conditions of up to $10 \mathrm{~m} / \mathrm{s}^{2}$ over a frequency range from $5 \mathrm{~Hz}$ to $150 \mathrm{~Hz}$.

g. Shock

The power supply is expected to function normally when exposed to shock conditions of up to $150 \mathrm{~m} / \mathrm{s}^{2}$.

h. Electrostatic discharge

The power supply is to function normally when exposed to electrostatic discharges that are not greater than $4 \mathrm{kV}$ contact, or $8 \mathrm{kV}$ air (IEC 61000-4-2).

The points of discharges are defined based on user access during normal operation.

i. Conducted disturbances induced by radio frequencies

The power supply is expected to function normally when exposed to disturbances conducted onto the input lines from radio frequency emissions from 0.15 to 80 $\mathrm{MHz}$ at $10 \mathrm{~V} / \mathrm{m}$.

j. $\quad$ Radiated radio frequency fields

The power supply shall function normally when exposed to radiated RF at frequencies from 80 to $1000 \mathrm{MHz}$ at $10 \mathrm{~V} / \mathrm{m}$

k. Voltage dips and short interruptions

The power supply's output shall remain acceptable when exposed to voltage dips and short interruptions according to the following table.

\begin{tabular}{|c|c|c|}
\hline $\begin{array}{c}\text { Voltage dips and short } \\
\text { interruptions } \\
\text { (\% of mains voltage) }\end{array}$ & $\begin{array}{c}\text { Duration } \\
\text { (ms) }\end{array}$ & No. Dips \\
\hline 100 & 20 & 1000 \\
\hline 60 & 20 & 1000 \\
\hline 30 & 20 & 1000 \\
\hline
\end{tabular}

l. Electrical fast transient (Burst) (IEC references 61000-4-4)

The power supply shall function normally when exposed to the following electrical fast transients on the mains input (severity level 4 - severe industrial environment):

\begin{tabular}{|c|c|}
\hline $\begin{array}{c}\text { Voltage Peak } \\
\text { KV }\end{array}$ & $\begin{array}{c}\text { Repetition Rate } \\
\mathbf{k H z}\end{array}$ \\
\hline 42 & 100 \\
\hline
\end{tabular}

m. Surge (IEC references 61000-4-5)

The power supply shall function normally when exposed to the following transients on the mains input: $1.2 / 50 \mu \mathrm{s}-8 / 20 \mu$ s combination waveform at an intensity of $42 \mathrm{kV}$.

4. Testing Protocol

a. Nominal environmental conditions

i. $20^{\circ} \mathrm{C} \pm 2{ }^{\circ} \mathrm{C}, 40-75 \% \mathrm{RH}$

ii. Ambient EM fields less than those that can cause interference.

b. Function

i. Connect the power supply to the line and the test load (the test load shall be equivalent to $100 \%$ of the stated maximum load for the selected unit). 
ii. Slowly reduce the input voltage in $5 \%$ increments until reaching the 15\% voltage and record Record the following information:

1. Output $D C$ voltage leveland output ripple voltage (ms-rms value)

2. Line perturbations

Line perturbations shall be within the following limits (Class A from NF EN 55022 standard):

\begin{tabular}{|c|c|c|}
\hline \multirow{2}{*}{$\begin{array}{c}\text { Frequency range } \\
(\mathrm{MHz})\end{array}$} & \multicolumn{2}{|c|}{ Limits $\quad \mathrm{dB}(\mu \mathrm{V})$} \\
\hline & Q - Peak & Average \\
\hline 0.15 to 0.50 & 79 & 66 \\
\hline 0.5 to 30 & 73 & 60 \\
\hline
\end{tabular}

iii. Slowly reduce the input voltage until reaching $-15 \%$ voltage and repeat step 4.b.ii.

iii.iv. Slowly return the input voltage to the nominal setting and note any unusual voltage spikes, if they occur.

$i \vee . v$. Slowly increase the input voltage in 5\% increments-until reaching the $+15 \%$ voltage and record the following information:repeat step 4.b.ii.

1. Output voltage level

2. Line perturbations

Line perturbations shall be within the following limits (Class $A$ from NF EN 55022 standard):

\begin{tabular}{|c|c|c|}
\hline \multirow{2}{*}{$\begin{array}{c}\text { Frequency range } \\
(\mathrm{MHz})\end{array}$} & \multicolumn{2}{|c|}{ Limits $\quad \mathrm{dB}(\mu \mathrm{V})$} \\
\hline & Q-Peak & Average \\
\hline 0.15 to 0.50 & 79 & 66 \\
\hline 0.5 to 30 & 73 & 60 \\
\hline
\end{tabular}

v. Repeat step iii.

vi. Repeat step 4.b.iv.

vii. Connect the power supply to a test load (the test load shall be equivalent to 50\% of the stated maximum load for the selected unit). Repeat step 4.b.ii.

viii. Disconnect the power supply from test load (no load). Repeat step 4.b.ii. Reconnect the power supply to $100 \%$ load.

$\forall$ i.ix. Perform the following test while measuring the output voltage to ensure stability (under full load). The test is based on IEC 61000-4-11. The time interval between two dips is equal to 1 second. Each dip must be generated for each phase angle which are: $0^{\circ}, 45^{\circ}, 90^{\circ}, 135^{\circ}, 180^{\circ}, 225^{\circ}$, $270^{\circ}$ and $315^{\circ}$.

\begin{tabular}{|c|c|c|}
\hline $\begin{array}{c}\text { Voltage dips and short } \\
\text { interruptions } \\
\text { (\% of mains voltage) }\end{array}$ & $\begin{array}{c}\text { Duration } \\
\text { (ms) }\end{array}$ & No. Dips \\
\hline 100 & 20 & 1000 \\
\hline 60 & 20 & 1000 \\
\hline 30 & 20 & 1000 \\
\hline
\end{tabular}


c. Temperature

The following temperature test is based on conditions stated in IEC 60721-4-3: Stationary use at weather protected locations - class 3K4, 60068-2-2 Bb/Bd: Dry heat, and 60068-2-1 Ab/Ad: Cold.

i. The power supply shall be placed in an environmental chamber and set up per step 4.b.i. The chamber shall then be stabilized at the reference conditions $\left(20^{\circ} \mathrm{C} \pm 2{ }^{\circ} \mathrm{C}, 40-75 \% \mathrm{RH}\right)$.

ii. The temperature is then reduced to $+5^{\circ} \mathrm{C}$ at a rate not exceeding $10^{\circ} \mathrm{C} / \mathrm{hr}$. Relative humidity levels should not exceed the reference range.

iii. The temperature shall be maintained for at least 16 hours once the power supply reaches equilibrium with the ambient temperature.

iv. After 16 hours of exposure, perform steps 4.b.ii through viii.

v. The temperature shall then be increased at a rate not exceeding $10^{\circ} \mathrm{C} / \mathrm{hr}$ to $+45^{\circ} \mathrm{C}$.

vi. Repeat steps 4.c.iii and 4.c.iv.

vii. After the high temperature exposure, return the temperature to the reference value and repeat steps 4.b.ii through 4.b.viii.

viii. Document all results and report any functional abnormalities to the appropriate task leader.

d. Humidity

The relative humidity (RH) test is based on protocol stated in IEC 60721-4-3: Stationary use at weather protected locations - class 3K4, and 60068-2-56 Cb: Damp heat. The conditions values stated were selected due to the possibility of equipment being used in environments that are similar to tropical conditions (+40 ${ }^{\circ} \mathrm{C}$ and 93\% (non-condensing).

i. The power supply shall be placed in an environmental chamber and set up per step 4.b.i. The chamber shall then be stabilized at the reference conditions $\left(20^{\circ} \mathrm{C} \pm 2{ }^{\circ} \mathrm{C}, 40-75 \% \mathrm{RH}\right)$.

ii. The temperature is then increased to $+40{ }^{\circ} \mathrm{C}$ at a rate not exceeding $10{ }^{\circ} \mathrm{C}$ /hr.

iii. After allowing time for the power supply to reach equilibrium with the ambient temperature, the $\mathrm{RH}$ level is increased to 93\% over a one-hour period.

iv. These conditions shall be maintained for 96 hours, during which the power supply is tested per steps 4.b.ii through 4.b.viviii at one12-hour intervals.

v. After 96 hours and the final function test, the ambient conditions shall be returned to the reference conditions at a rate not exceeding $10^{\circ} \mathrm{C} / \mathrm{hr}$.

vi. Once the power supply reaches thermal equilibrium, its function shall be tested per 4.b.ii through 4.b.i¥ix.

vii. Document all results and report any functional abnormalities to the appropriate task leader.

e. Condensation

Due to the possibility of condensation exposure, the following test is required. The test is based on IEC 60068-2-30 has different requirements. 3K4 recommends Db variant 2 which is $+30{ }^{\circ} \mathrm{C}, 90-100 \% \mathrm{RH}$ for 2 cycles)

i. Prior to exposure, verify that the power supply is functioning properly. Do not proceed if the unit is not functioning properly. 
ii. Increase the RH level to 95\%.

iii. The temperature of the chamber shall then be raised to $+30{ }^{\circ} \mathrm{C}$ over a $3 \mathrm{~h}$ \pm 30 min time interval. During this period, the relative humidity shall be not less than 95\%, except during the last 15 min when it may be from 90 to $95 \%$. Condensation should occur on the power supply during this temperature-rise period.

iv. The temperature shall then be maintained within the prescribed limits for the upper temperature $\left( \pm 2^{\circ} \mathrm{C}\right)$ until $12 \mathrm{~h} \pm 30$ min from the start of the cycle. During this period, the relative humidity shall be $93 \pm 3 \%$ except for the first and last 15 min when it may be between $90 \%$ and $100 \%$.

v. The temperature shall then be lowered to $25^{\circ} \mathrm{C} \pm 3{ }^{\circ} \mathrm{C}$ over a period of 3 $\mathrm{h}$ while maintaining the $\mathrm{RH}$ level at $93 \pm 3 \%$.

vi. The temperature shall then be maintained at $25^{\circ} \mathrm{C} \pm 3{ }^{\circ} \mathrm{C}$ with a relative humidity of not less than $95 \%$ until the $24 \mathrm{~h}$ cycle is completed.

vii. This entire process shall be repeated once more.

viii. Prior to temperature change steps, the power supply's function shall be tested per steps 4.b.ii through 4.b.viviii and results recorded.

ix. Once the second cycle is complete, reduce the temperature and $\mathrm{RH}$ level to the reference conditions and re-test the ehargeepower supply per steps 4.b.ii through 4.b.viix.

$\mathrm{x}$. After the final test, inspect the power supply for moisture ingress and corrosion. Ensure that power is removed before inspection.

xi. Document all results and report any functional abnormalities to the appropriate task leader.

f. Vibration

The vibration test is based on conditions stated in IEC 60721-3-3, Class 3M4 and 60068-2-6 Fc: Vibration sinusoidal. The power supply shall be functionally tested per step 4.b prior to each exposure,-during exposure, and afterthe output voltage monitored during exposure. After each axis is finished. the power supply shall be functionally tested per step 4.b.

i. Vibration parameters are as follows:

1. Acceleration $=10 \mathrm{~m} / \mathrm{s}^{2}$

2. Frequency Range $=5 \mathrm{~Hz}$ to $150 \mathrm{~Hz}$

3. Axes $=3$

4. Sweep Cycles $=10$ (about 2 hours per axis)

ii. Document all results and report any functional abnormalities to the appropriate task leader.

g. Shock

The shock (bump) test is based on conditions stated in IEC 60721-3-3, Class 3M4 and 60068-2-29 Eb: Bump. The power supply shall be functionally tested per step 4.b prior to each exposure and the output voltage monitored during exposure. After each axis is finished the power supply shall be functionally tested per step 4.b.

g.The power supply shall be functionally tested per step $4 . b$ prior to each exposure, during exposure, and after each axis is finished.

i. Shock test parameters are as follows:

1. Peak Acceleration $=150 \mathrm{~m} / \mathrm{s}^{2}$

2. Duration $=6 \mathrm{~ms}$

3. Number of shocks/bumps $=100$ in each direction 


\section{Direction $=3$}

ii. Document all results and report any functional abnormalities to the appropriate task leader.

h. Radiated emissions

RF emissions shall not exceed the levels stated below. Tests shall be performed using a SAS antenna or equivalent device and a spectrum analyzer. Emissions measurements shall be made with the antenna placed one meter from the center of the power supply. Measurements shall be made with the power supply functioning with full line voltage and maximum load.

\begin{tabular}{|c|c|}
\hline Frequency range $\mathrm{MHz}$ & $\begin{array}{c}\text { Limits } \mathrm{dB}(\mu \mathrm{V}) \\
\mathrm{Q}-\text { Peak }\end{array}$ \\
\hline 30 to 230 & 40 \\
\hline 230 to 1000 & 47 \\
\hline
\end{tabular}

i. Electrostatic discharge

The power supply shall be exposed to a series of $4 \mathrm{kV}$ contact discharges and 8 $\mathrm{kV}$ air discharges (IEC 61000-4-2). The discharge points shall be selected based on possible user access during normal operation. Conductive surfaces will require contact discharge exposures; non-conductive surfaces will be exposed to air discharges.

i. Prior to testing, the power supply shall be functioning normally and eperated tested based on 4.b.ii through 4.b.vi.ix.

ii. During exposures, the output voltage and voltage waveform shall be monitored. according to section 4.6 .

iii. After the test, the power supply shall be tested per 4.b.ii through viix.

iv. Document all results and report any functional abnormalities to the appropriate task leader.

j. $\quad$ Conducted disturbances induced by radio frequencies

The fields shown below will be applied to the input lines through a coupling/decoupling network. During exposure, the power supply willoutput voltage and voltage waveform shall be functionally tested using guidance found in 4.b.i through vimonitored.

Document all results and report any functional abnormalities to the appropriate task leader.

\begin{tabular}{|c|c|c|c|c|}
\hline $\begin{array}{c}\text { Frequency } \\
\text { Range } \\
\text { (MHz) }\end{array}$ & $\begin{array}{c}\text { Field } \\
\text { Strength } \\
\text { (V/m) }\end{array}$ & $\begin{array}{c}\% \mathrm{AM} \\
1 \mathrm{kHz}\end{array}$ & $\begin{array}{c}\text { Dwell Time } \\
\text { (s) }\end{array}$ & $\begin{array}{c}\text { Frequency } \\
\text { step size }\end{array}$ \\
\hline 0.15 to 80 & 10 & 80 & 5 & $\begin{array}{c}1 \% \text { of the } \\
\text { fundamental }\end{array}$ \\
\hline
\end{tabular}

k. Radiated radio frequency fields

The power supply shall be exposed to the fields shown below. During exposure, the power supply willoutput voltage and voltage waveform shall be functionally tested using guidance found in $4 . b$.i through vimonitored.

Document all results and report any functional abnormalities to the appropriate task leader. The test procedure for the following test must be in accordance with IEC 61000-4-3, class 3. 


\begin{tabular}{|c|c|c|c|c|}
\hline $\begin{array}{c}\text { Frequency } \\
\text { Range } \\
\text { (MHz) }\end{array}$ & $\begin{array}{c}\text { Field } \\
\text { Strength } \\
\text { (V/m) }\end{array}$ & $\begin{array}{c}\% \mathrm{AM} \\
1 \mathrm{kHz}\end{array}$ & $\begin{array}{c}\text { Dwell Time } \\
\text { (s) }\end{array}$ & $\begin{array}{c}\text { Frequency } \\
\text { step size }\end{array}$ \\
\hline 80 to 1000 & 10 & 80 & 5 & $\begin{array}{c}1 \% \text { of the } \\
\text { fundamental }\end{array}$ \\
\hline
\end{tabular}

1. Electrical fast transient (Burst) (IEC references 61000-4-4)

i. Electrical fast transients (bursts) shall be applied to the mains supply terminals via a coupling/decoupling network, or equivalent equipment. The repetition rate should not exceed once per minute.

ii. Expose the power supply to a series of transients with a minimum time between each of one minute.

iii. Each transient shall consist of a ring wave with a peak voltage of $2 \mathrm{kV}$ and a repetition rate of $100 \mathrm{kHz}$.

iv. Monitor the output voltage of the power supply throughout the test.

v. Document all results and report any functional abnormalities to the appropriate task leader.

m. Surge tests (IEC references 61000-4-5)

i. Pulses shall be applied to the mains supply terminals via a coupling/decoupling network, or equivalent equipment. The repetition rate should not exceed once per minute.

ii. Expose the power supply to ten pulses with a minimum time between surges of one minute.

iii. Each pulse shall consist of a combination wave $(1.2 / 50 \mu \mathrm{s}-8 / 20 \mu \mathrm{s})$ at an intensity of $42 \mathrm{kV}$.

iv. Monitor the output voltage of the power supply throughout the test.

v. Document all results and report any functional abnormalities to the appropriate task leader. 


\section{APPENDIX C}

IAEA Performance Specification Uninterruptible Power Supply (UPS) 


\section{IAEA Performance Specification \\ Uninterruptible Power Supply (UPS)}

\section{Background}

Most IAEA safeguards systems incorporate UPS devices to ensure that power quality is maintained for the attached system and to permit an acceptable level of system functionality if power is lost. An UPS may be used in different environments, which do not include any unexpected ionizing radiation fields.

The load for testing purposes shall be $75 \%$ of the stated maximum linear load for the selected UPS unit.

\section{Requirements for Use}

1. Functional

a. Input power

i. Frequency

ii. Voltage

1. 50 or $60 \mathrm{~Hz}( \pm 5 \%)$

1. Nominal voltage is 100 to $230 \mathrm{VAC}$ ( $\pm 15 \%)$, or selectable between 100, 115, and 230 VAC.

iii. Current

1. The input current should be limited. Defined values are dependent on the specific application.

b. Output power

i. Transfer time

1. $<4 \mathrm{~ms}$.

ii. Transfer Activation

1. Nominal voltage $\pm 15 \%$.

iii. Stability

1. Voltage to be within $\pm 5 \%$.

2. Frequency $\pm 0.5 \%$ of nominal $(50$ or $60 \mathrm{~Hz})$.

iv. Sine wave quality (distortion)

1. $<3 \%$ at maximum linear load.

v. Line perturbations

Line perturbations on the output power shall not exceed the following values.

\begin{tabular}{|c|cc|}
\hline \multirow{2}{*}{$\begin{array}{c}\text { Frequency } \\
\text { range } \mathrm{MHz}\end{array}$} & Limits & $\mathrm{dB}(\mu \mathrm{V})$ \\
\cline { 2 - 3 } & $\mathrm{Q}-$ Peak & Average \\
\hline 0.15 to 0.50 & 79 & 66 \\
\hline 0.5 to 30 & 73 & 60 \\
\hline
\end{tabular}

c. Battery lifetime

i. Battery powered operation lifetime is not defined. Selection is based on the requirements of the individual IAEA system.

ii. Battery recharge time is not defined. Selection is based on expected use. 
d. Electrical safety requirements

The UPS shall meet appropriate electrical safety requirements. As a minimum, the unit shall meet appropriate ÖVE (Österreichischer Verein Der Elektrotechnik - Austrian Electrotechnical Association) requirements. The unit should also meet the requirements of the country of use.

e. Data communication

Existing systems use RS-232, which based on a market search is the most common communication format. USB is also available as well as 10baseT. The suggested requirement is "As a minimum, the UPS shall have the ability to provide an indication when a loss of mains voltage occurs. The communication format shall be RS-232. Other methods should be available such as USB or Ethernet.

f. Radiated emissions

Radiation emissions shall be less than the values shown below when measured at a distance of 1 meter (values are based on 10 meters and are from NF EN 55022 standard).

\begin{tabular}{|c|c|}
\hline Frequency range $\mathrm{MHz}$ & $\begin{array}{c}\text { Limits dB }(\mu \mathrm{V}) \\
\mathrm{Q}-\mathrm{Peak}\end{array}$ \\
\hline 30 to 230 & 40 \\
\hline 230 to 1000 & 47 \\
\hline
\end{tabular}

2. Mechanical design

a. Must be able to be used in a 19" wide rack and be not more than 60-65 cm deep.

b. $<60 \mathrm{~kg}$, excluding batteries

3. Expected environmental conditions

a. Ambient temperature +5 to $+45^{\circ} \mathrm{C}$ (based on 60721-4-3, class 3K4)

b. Relative humidity $+40{ }^{\circ} \mathrm{C}$ and $93 \%$ (3K4 shows +30 and $93 \%$ for 96 hours). With required inspection, this test could cover the corrosion requirement.

c. Condensation UPS should be able to operate when exposed to a condensing atmosphere.

d. Atmospheric pressure No requirement.

e. Dust and splash water The IAEA cabinet is required to meet IP56 (dust proof and spray proof, respectively); therefore no requirement is stated for individual components. (EN 60529/IEC 529).

f. Vibration

The UPS is expected to function normally when exposed to vibrations conditions of up to $10 \mathrm{~m} / \mathrm{s}^{2}$ over a frequency range from $5 \mathrm{~Hz}$ to $150 \mathrm{~Hz}$.

g. Shock

The UPS is expected to function normally when exposed to shock conditions of up to $150 \mathrm{~m} / \mathrm{s}^{2}$.

h. Electrostatic discharge

The UPS is to function normally when exposed to electrostatic discharges that are not greater than $4 \mathrm{kV}$ contact, or $8 \mathrm{kV}$ air (IEC 61000-4-2). The points of discharges are defined based on user access during normal operation. 
i. Conducted disturbances induced by radio frequencies

The UPS is expected to function normally when exposed to disturbances

conducted onto the input lines from radio frequency emissions from 0.15 to 80

$\mathrm{MHz}$ at $10 \mathrm{~V} / \mathrm{m}$.

j. $\quad$ Radiated radio frequency fields

The UPS shall function normally when exposed to radiated RF at frequencies from 80 to $1000 \mathrm{MHz}$ at $10 \mathrm{~V} / \mathrm{m}$

k. Voltage dips and short interruptions (IEC 61000-4-11)

The UPS output shall remain acceptable when exposed to voltage dips and short interruptions according to the following table.

\begin{tabular}{|c|c|c|}
\hline $\begin{array}{c}\text { Voltage dips and short } \\
\text { interruptions } \\
\text { (\% of mains voltage) }\end{array}$ & $\begin{array}{c}\text { Duration } \\
\text { (ms) }\end{array}$ & No. Dips \\
\hline 100 & 20 & 1000 \\
\hline 60 & 20 & 1000 \\
\hline 30 & 20 & 1000 \\
\hline
\end{tabular}

1. Electrical fast transient (Burst) (IEC references 61000-4-4)

The UPS shall function normally when exposed to the following electrical fast transients on the mains input (severity level 4 - severe industrial environment):

\begin{tabular}{|c|c|}
\hline $\begin{array}{c}\text { Voltage Peak } \\
\text { KV }\end{array}$ & $\begin{array}{c}\text { Repetition Rate } \\
\mathbf{k H z}\end{array}$ \\
\hline 42 & 100 \\
\hline
\end{tabular}

m. Surge (IEC references 61000-4-5)

The UPS shall function normally when exposed to the following transients on the mains input: 1.2/50 $\mu \mathrm{s}-8 / 20 \mu \mathrm{s}$ combination waveform at an intensity of $4-2 \mathrm{kV}$.

4. Testing Protocol

a. Nominal environmental conditions

i. $20 \pm 2{ }^{\circ} \mathrm{C}, 40-75 \% \mathrm{RH}$

ii. Ambient EM fields less than those that can cause interference.

b. Function

i. Connect the UPS to the appropriate mains source and the test load. The test load shall be equivalent to $75 \%$ of the stated maximum linear load for the selected UPS unit.

ii. Slowly reduce the mains voltage until the UPS activates (nominal $15 \%$ ) and record the following information (some UPS devices are always on and there is no transfer time):

1. Mains voltage at transfer (within $\pm 15 \%$ of nominal voltage)

2. Response/transfer time ( $<4 \mathrm{~ms})$

3. Waveform (distortion shall be $<3 \%$ of that at linear load)

4. Output voltage level from onset of transfer to stabilization (within $\pm 5 \%$ of required output)

5. Frequency $( \pm 0.5 \%$ of nominal -50 or $60 \mathrm{~Hz})$

6. Line perturbations

Line perturbations shall be within the following limits (Class A from NF EN 55022 standard): 


\begin{tabular}{|c|c|c|}
\hline \multirow{2}{*}{$\begin{array}{c}\text { Frequency range } \\
(\mathrm{MHz})\end{array}$} & \multicolumn{2}{|c|}{ Limits $\quad \mathrm{DB}(\mu \mathrm{V})$} \\
\hline & Q - Peak & Average \\
\hline 0.15 to 0.50 & 79 & 66 \\
\hline 0.5 to 30 & 73 & 60 \\
\hline
\end{tabular}

iii. Slowly return the mains voltage to the nominal setting and record when the UPS transfers function back to the mains. Note any unusual voltage spikes or sinusoidal changes, if they occur.

iv. Slowly increase the mains voltage until the UPS activates (nominal + 15\%) and record the following information:

1. Mains voltage at transfer

2. Response/transfer time

3. Waveform

4. Output voltage level from onset of transfer to stabilization

v. Repeat step iii.

vi. Perform the following test while measuring the output voltage to ensure stability. The test is based on IEC 61000-4-11. The time interval between two dips is equal to 1 second. Each dip must be generated for each phase angle which are: $0^{\circ}, 45^{\circ}, 90^{\circ}, 135^{\circ}, 180^{\circ}, 225^{\circ}, 270^{\circ}$ and $315^{\circ}$.

\begin{tabular}{|c|c|c|}
\hline $\begin{array}{c}\text { Voltage dips and short } \\
\text { interruptions } \\
\text { (\% of mains voltage) }\end{array}$ & $\begin{array}{c}\text { Duration } \\
\text { (ms) }\end{array}$ & No. Dips \\
\hline 100 & 20 & 1000 \\
\hline 60 & 20 & 1000 \\
\hline 30 & 20 & 1000 \\
\hline
\end{tabular}

c. Temperature

The following temperature test is based on conditions stated in IEC 60721-4-3: Stationary use at weather protected locations - class 3K4, 60068-2-2 Bb/Bd: Dry heat, and 60068-2-1 Ab/Ad: Cold.

i. The UPS shall be placed in an environmental chamber. The chamber shall then be stabilized at the reference conditions $\left(20^{\circ} \mathrm{C} \pm 2{ }^{\circ} \mathrm{C}, 40-75 \%\right.$ $\mathrm{RH})$.

ii. The temperature is then reduced to $+5{ }^{\circ} \mathrm{C}$ at a rate not exceeding $10^{\circ} \mathrm{C} / \mathrm{hr}$. Relative humidity levels should not exceed the reference range.

iii. The temperature shall be maintained for at least 16 hours once the UPS reaches equilibrium with the ambient temperature.

iv. After 16 hours of exposure, perform steps 4.b.ii through vi.

v. The temperature shall then be increased at a rate not exceeding $10^{\circ} \mathrm{C}$ $\mathrm{C} / \mathrm{hr}$ to $+45^{\circ} \mathrm{C}$.

vi. Repeat steps 4.c.iii and iv.

vii. After the high temperature exposure, return the temperature to the reference level and repeat steps 4.b.ii through 4.b.vi.

viii. Document all results and report any functional abnormalities to the appropriate task leader. 


\section{d. Humidity}

The relative humidity (RH) test is based on protocol stated in IEC 60721-4-3: Stationary use at weather protected locations - class 3K4, and 60068-2-56 Cb: Damp heat. The conditions values stated were selected due to the possibility of equipment being used in environments that are similar to tropical conditions ( +40 ${ }^{\circ} \mathrm{C}$ and 93\% (non-condensing).

i. Place the UPS in an environmental chamber. The chamber is stabilized at the reference conditions $\left(20^{\circ} \mathrm{C} \pm 2{ }^{\circ} \mathrm{C}, 40-75 \% \mathrm{RH}\right)$.

ii. The temperature is then increased to $+40{ }^{\circ} \mathrm{C}$ at a rate not exceeding $10{ }^{\circ} \mathrm{C}$ /hr.

iii. After allowing time for the UPS to reach equilibrium with the ambient temperature, the RH level is increased to $93 \%$ over a one-hour period. Note - the UPS may generate heat during normal use. If this is the case, equilibrium shall be assumed at the completion of the temperature change ramp.

iv. These conditions shall be maintained for 96 hours, during which the UPS is functionally tested (4.b.ii through vi) at one12-hour intervals.

v. After 96 hours and the final function test, the ambient conditions shall then be returned to the reference conditions at a rate not exceeding $10^{\circ} \mathrm{C}$ /hr.

vi. Once the UPS reaches thermal equilibrium, its function shall be tested (4.b.ii through vi).

vii. Document all results and report any functional abnormalities to the appropriate task leader.

e. Condensation

Due to the possibility of condensation exposure, the following test is required. The test is based on IEC 60068-2-30 has different requirements. 3K4 recommends Db variant 2 which is $+30,90-100 \%$ RH for 2 cycles)

i. Prior to exposure, verify that the UPS is functioning properly. Do not proceed if the unit is not functioning properly.

ii. Increase the $\mathrm{RH}$ level to $95 \%$.

iii. The temperature of the chamber shall then be raised to $+30{ }^{\circ} \mathrm{C}$ over a $3 \mathrm{~h}$ \pm 30 min time interval. During this period, the relative humidity shall be not less than 95\%, except during the last 15 min when it may be from 90 to $95 \%$. Condensation should occur on the specimen during this temperature-rise period.

iv. The temperature shall then be maintained within the prescribed limits for the upper temperature $\left( \pm 2^{\circ} \mathrm{C}\right)$ until $12 \mathrm{~h} \pm 30 \mathrm{~min}$ from the start of the cycle. During this period, the relative humidity shall be $93 \pm 3 \%$ except for the first and last 15 min when it may be between $90 \%$ and $100 \%$.

v. The temperature shall then be lowered to $25 \pm 3{ }^{\circ} \mathrm{C}$ over a period of $3 \mathrm{~h}$ while maintaining the $\mathrm{RH}$ level at $93 \pm 3 \%$.

vi. The temperature shall then be maintained at $25 \pm 3{ }^{\circ} \mathrm{C}$ with a relative humidity of not less than $95 \%$ until the $24 \mathrm{~h}$ cycle is completed.

vii. This entire process shall be repeated once more.

viii. Prior to temperature change steps, the UPS function shall be tested and results recorded (4.b.ii through vi).

ix. Once the second cycle is complete, reduce the temperature and $\mathrm{RH}$ level to the reference conditions and re-test the UPS (4.b.ii through vi). 
x. After the final test, inspect the UPS for moisture ingress and corrosion. Ensure that power is removed before inspection.

xi. Document all results and report any functional abnormalities to the appropriate task leader.

f. Vibration

The vibration test is based on conditions stated in IEC 60721-3-3, Class 3M4 and 60068-2-6 Fc: Vibration sinusoidal. The UPS shall be functionally tested prior to each exposure,during exposure, and after each axis is finished. The output voltage shall be monitored during exposure.

i. Vibration parameters are as follows:

1. Acceleration $=10 \mathrm{~m} / \mathrm{s}^{2}$

2. Frequency Range $=5 \mathrm{~Hz}$ to $150 \mathrm{~Hz}$

3. Axes $=3$

4. Sweep Cycles $=10$ (about 2 hours per axis)

ii. Document all results and report any functional abnormalities to the appropriate task leader.

g. Shock

The shock (bump) test is based on conditions stated in IEC 60721-3-3, Class 3M4 and 60068-2-29 Eb: Bump. The UPS shall be functionally tested prior to each exposure,-during exposure, and after each axis is finished. The output voltage shall be monitored during exposure.

i. Shock test parameters are as follows:

1. Peak Acceleration $=150 \mathrm{~m} / \mathrm{s}^{2}$

2. Duration $=6 \mathrm{~ms}$

3. Number of shocks/bumps $=100$ in each direction

4. Direction $=3$

ii. Document all results and report any functional abnormalities to the appropriate task leader.

h. Radiated emissions

RF emissions shall not exceed the levels stated below. Tests shall be performed using a SAS antenna or equivalent device and a spectrum analyzer. Emissions measurements shall be made with the antenna placed one meter from the center of the UPS. Measurements shall be made with the UPS monitoring line power (pass-through mode) and with the UPS functioning (inverter mode).

\begin{tabular}{|c|c|}
\hline Frequency range $\mathrm{MHz}$ & $\begin{array}{c}\text { Limits } \mathrm{dB}(\mu \mathrm{V}) \\
\mathrm{Q}-\text { Peak }\end{array}$ \\
\hline 30 to 230 & 40 \\
\hline 230 to 1000 & 47 \\
\hline
\end{tabular}

i. Electrostatic discharge

The UPS shall be exposed to a series of $4 \mathrm{kV}$ contact discharges and $8 \mathrm{kV}$ air discharges (IEC 61000-4-2). The discharge points shall be selected based on possible user access during normal operation. Conductive surfaces will require contact discharge exposures; non-conductive surfaces will be exposed to air discharges. 
i. Prior to testing, the UPS shall be functioning normally and exercised to ensure proper operation.

ii. During exposures, the output line voltage and voltage waveform shall be monitored-according to section 4.b.

iii. After the test, the UPS shall be tested per 4.b.ii through vi.

iv. Document all results and report any functional abnormalities to the appropriate task leader.

j. Conducted disturbances induced by radio frequencies

The fields shown below will be applied to the mains through a coupling/decoupling network. During exposure, the UPS will be functionally tested using guidance found in 4.b.ii through vioutput voltage and voltage waveform shall be monitored. Testing shall be done in both the pass-through mode and inverter mode.

Document all results and report any functional abnormalities to the appropriate task leader.

\begin{tabular}{|c|c|c|c|c|}
\hline $\begin{array}{c}\text { Frequency } \\
\text { Range } \\
(\mathrm{MHz})\end{array}$ & $\begin{array}{c}\text { Field } \\
\text { Strength } \\
(\mathrm{V} / \mathrm{m})\end{array}$ & $\begin{array}{c}\% \mathrm{AM} \\
1 \mathrm{kHz}\end{array}$ & $\begin{array}{c}\text { Dwell Time } \\
\text { (s) }\end{array}$ & $\begin{array}{c}\text { Frequency } \\
\text { step size }\end{array}$ \\
\hline 0.15 to 80 & 10 & 80 & 5 & $\begin{array}{c}1 \% \text { of the } \\
\text { fundamental }\end{array}$ \\
\hline
\end{tabular}

k. Radiated radio frequency fields

The UPS shall be exposed to the fields shown below. During exposure, the UPS will be functionally tested using guidance found in 4 .b.ii through vioutput voltage and voltage waveform shall be monitored. Testing shall be done in both the pass-through mode and the inverter mode.

Document all results and report any functional abnormalities to the appropriate task leader. The test procedure for the following test must be in accordance with IEC 61000-4-3, class 3.

\begin{tabular}{|c|c|c|c|c|}
\hline $\begin{array}{c}\text { Frequency } \\
\text { Range } \\
\text { (MHz) }\end{array}$ & $\begin{array}{c}\text { Field } \\
\text { Strength } \\
\text { (V/m) }\end{array}$ & $\begin{array}{c}\% \mathrm{AM} \\
1 \mathrm{kHz}\end{array}$ & $\begin{array}{c}\text { Dwell Time } \\
\text { (s) }\end{array}$ & $\begin{array}{c}\text { Frequency } \\
\text { step size }\end{array}$ \\
\hline 80 to 1000 & 10 & 80 & 5 & $\begin{array}{c}1 \% \text { of the } \\
\text { fundamental }\end{array}$ \\
\hline
\end{tabular}

1. Electrical fast transient (Burst) (IEC references 61000-4-4)

i. Electrical fast transients (bursts) shall be applied to the mains supply terminals via a coupling/decoupling network, or equivalent equipment. The repetition rate should not exceed once per minute.

ii. Expose the UPS to a series of transients with a minimum time between each of one minute.

iii. Each transient shall consist of a ring wave with a peak voltage of $2 \mathrm{kV}$ and a repetition rate of $100 \mathrm{kHz}$.

iv. Monitor the output voltage of the UPS throughout the test.

v. Document all results and report any functional abnormalities to the appropriate task leader. 
m. Surge tests (IEC references 61000-4-5)

i. Pulses shall be applied to the mains supply terminals via a coupling/decoupling network, or equivalent equipment. The repetition rate should not exceed once per minute.

ii. Expose the UPS to ten pulses with a minimum time between surges of one minute.

iii. Each pulse shall consist of a combination wave $(1.2 / 50 \mu \mathrm{s}-8 / 20 \mu \mathrm{s})$ at an intensity of $4-2 \mathrm{kV}$.

iv. Monitor the output voltage of the UPS throughout the test.

v. Document all results and report any functional abnormalities to the appropriate task leader. 
APPENDIX D

Test Results 


\section{TEST RESULTS \\ FOR \\ BATTERY}

\begin{tabular}{|c|c|c|c|c|c|c|c|c|}
\hline & \multicolumn{4}{|c|}{ Discharge Time (Minutes) } & \multicolumn{4}{|c|}{ Charge Time (Minutes) } \\
\hline & 20 Deg C & 5 Deg C & 45 Deg C & 20 Deg C Repeat & 20 Deg C & 5 Deg C & 45 Deg C & 20 Deg C Repeat \\
\hline Battery A & 102 & 92 & 127 & 111 & 257 & 278 & 358 & 269 \\
\hline Battery B & 182 & 200 & 165 & 190 & 585 & 522 & 393 & 487 \\
\hline Battery C & 338 & 339 & 371 & 375 & 349 & 311 & 453 & No Data \\
\hline
\end{tabular}

Battery A = Sonnenschein A200 ( 60Ah)

Battery B = Sonnenschein A500 (110Ah)

Battery C = Panasonic LC-R123R4 (3.4Ah) 


\section{Battery A = Sonnenschein A200 ( 60Ah}

60Ah Battery Discharge at 20C

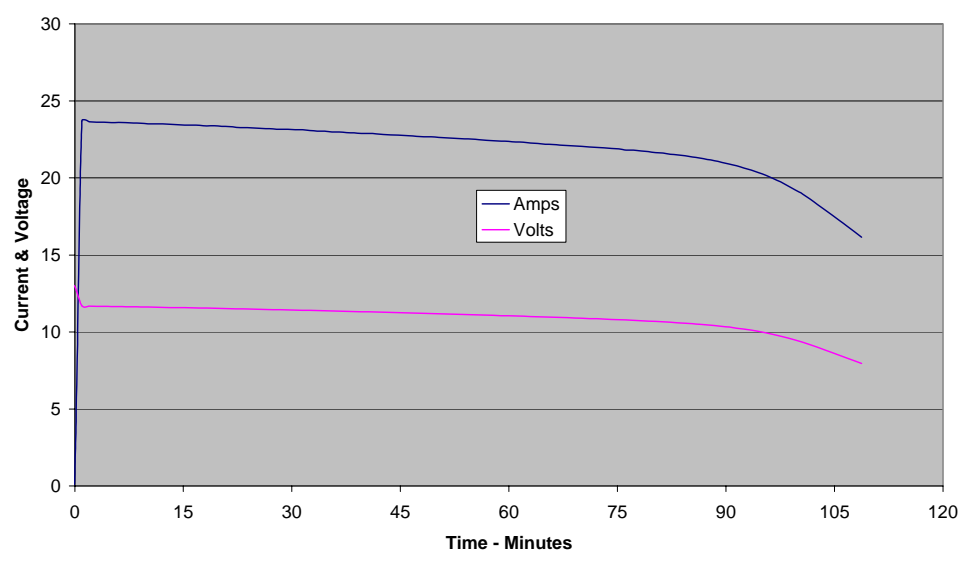

60Ah Battery Discharge Waveforms

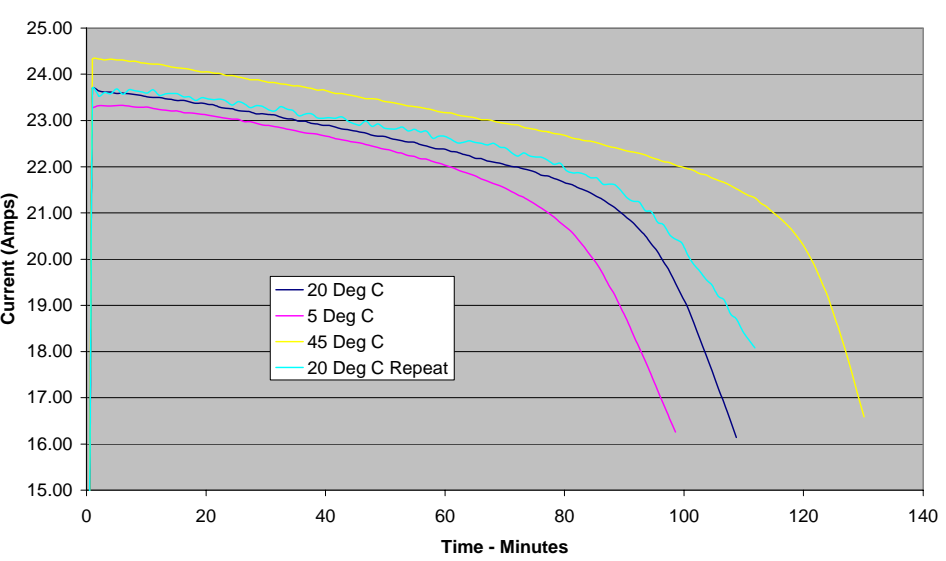

60Ah Battery Charge at 20C

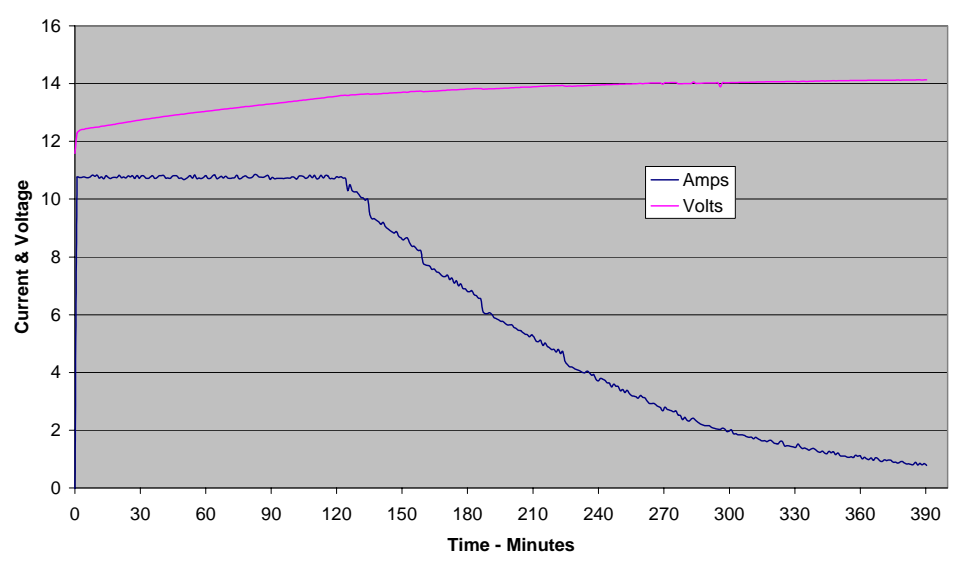

60Ah Battery Charge Waveforms

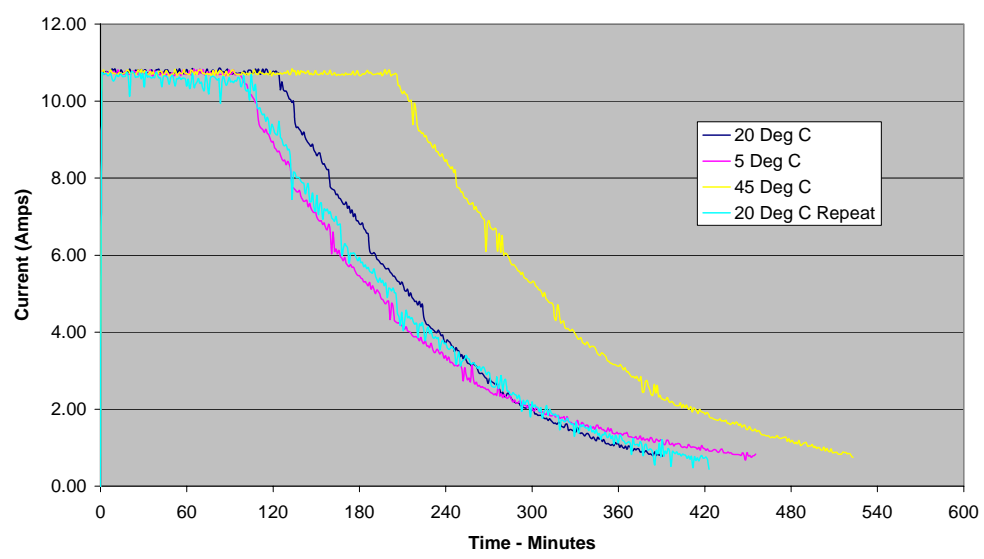




\section{Battery B = Sonnenschein A500 (110Ah)}

110Ah Battery Discharge at 20C

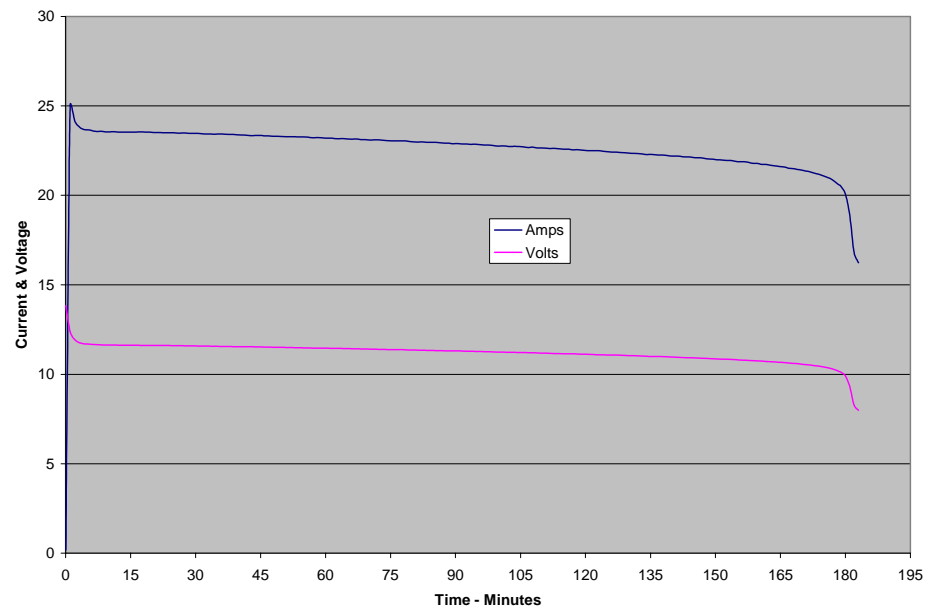

110Ah Battery Discharge Waveforms

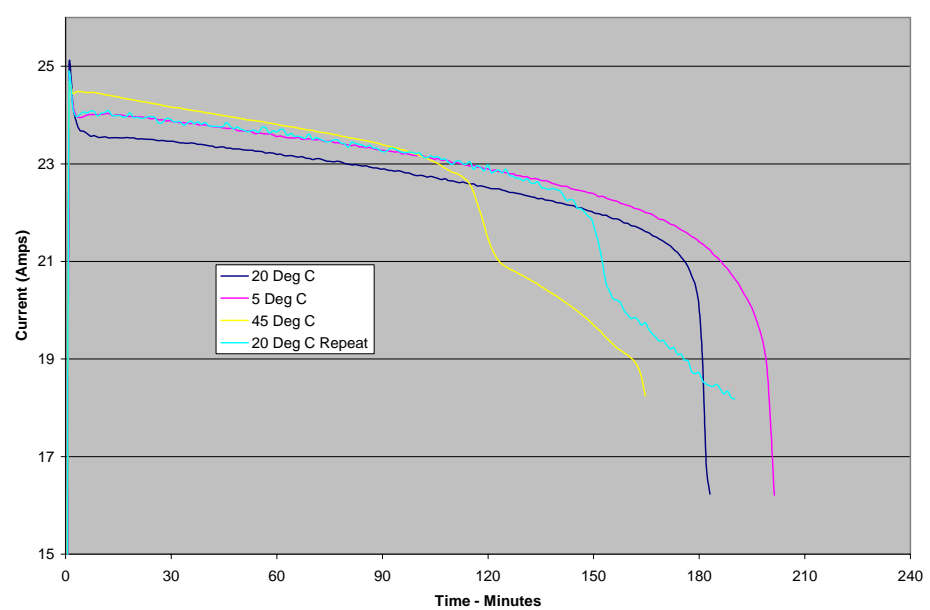

110Ah Battery Charge at 20C

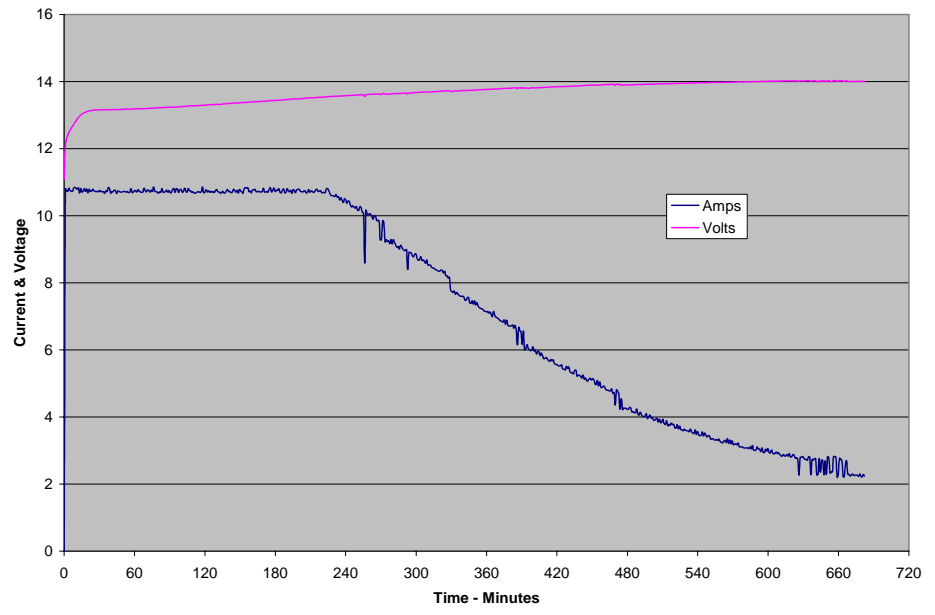

110Ah Battery Charge Waveforms

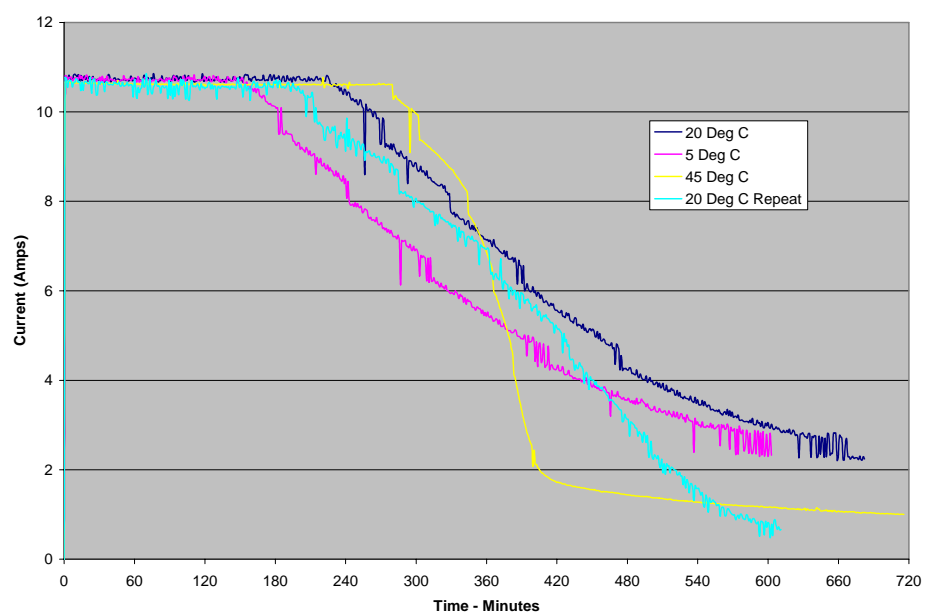




\section{Battery C = Panasonic LC-R123R4 (3.4Ah)}

3.4 Ah Battery Discharge at 20C

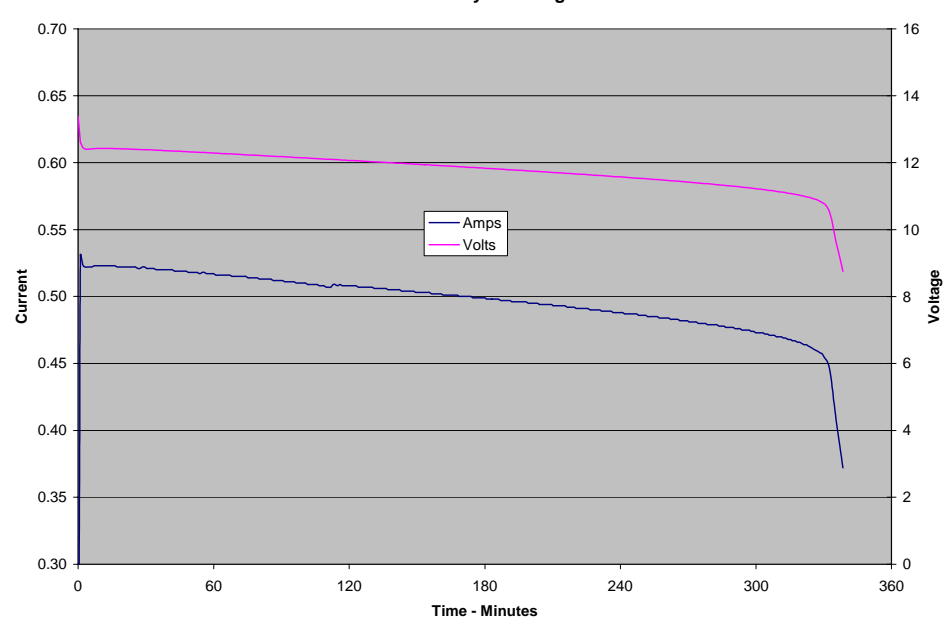

3.4 Ah Battery Discharge Current

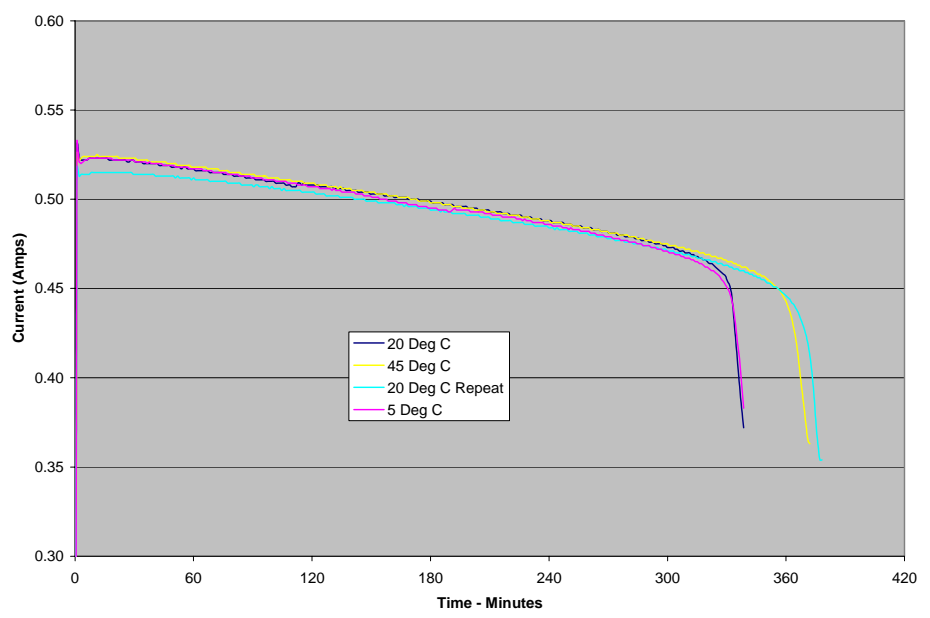

3.4 Ah Battery Charge at 20C

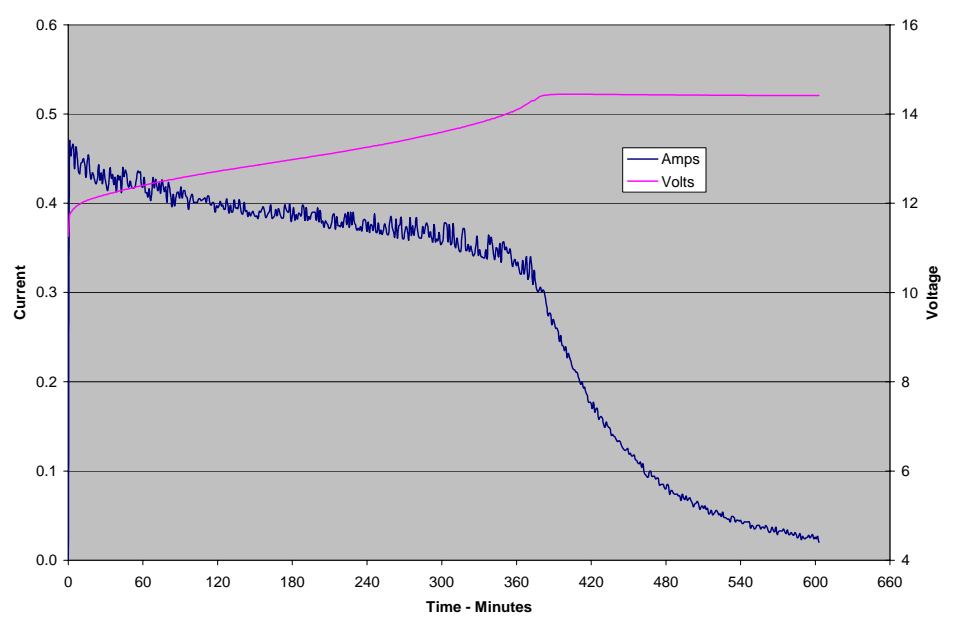

3.4 Ah Battery Charge Current

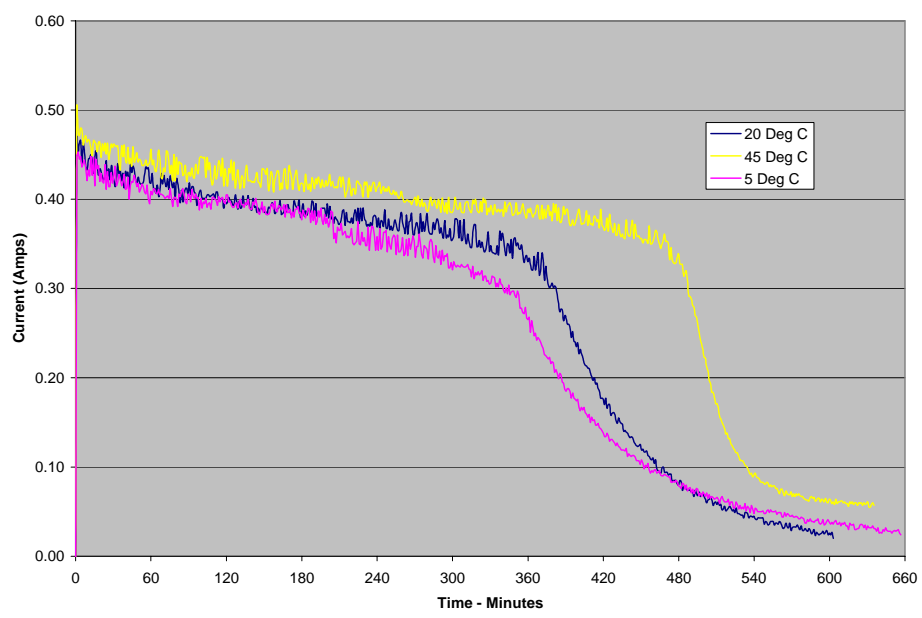




\section{TEST RESULTS}

\section{FOR}

LAMBDA POWER SUPPLY

EWS25-18 18 VOLTS

D - 5 


\section{Testing Data - Lambda PS EWS25-18 18volts}

Nominal Environmental Conditions (20 Deg C)

\begin{tabular}{|c|c|c|c|c|c|c|c|c|c|}
\hline \multicolumn{2}{|c|}{ Date/Time: 9/12/03 3:00 PM } & \multicolumn{2}{|c|}{ Full Load=1.5amps } & \multicolumn{2}{|c|}{ 19.9 Deg C } & \multicolumn{2}{|c|}{$46.0 \% \mathrm{RH}$} & $75 \%$ Load & No Load \\
\hline & Normal Voltage & Voltage $-5 \%$ & Voltage $-10 \%$ & Voltage $-15 \%$ & Voltage $+5 \%$ & Voltage $+10 \%$ & Voltage $+15 \%$ & $1.125 \mathrm{amps}$ & 0 amps \\
\hline Input (vac) & 230.0 & 218.5 & 207.0 & 195.5 & 241.5 & 253.0 & 263.7 & 230.0 & 230.0 \\
\hline Dutput (vdc) & 17.973 & 17.973 & 17.973 & 17.973 & 17.973 & 17.973 & 17.973 & Not Meas. & Not Meas. \\
\hline pple (mvac) & 8.0 & 8.1 & 7.8 & 7.9 & 7.9 & 7.8 & 8.1 & Not Meas. & Not Meas. \\
\hline
\end{tabular}

Comments:

Condensation Conditions

\begin{tabular}{|c|c|c|c|c|c|c|c|c|c|}
\hline & & & Condensatı & n Condition & & & & & \\
\hline Date/Time: & //13/03 9:00 AM & Full Load=1.5a & & 29 & Deg C & 92.3 & $\% \mathrm{RH}$ & $75 \%$ Load & No Load \\
\hline & Normal Voltage & Voltage $-5 \%$ & Voltage -10\% & Voltage -15\% & Voltage $+5 \%$ & Voltage $+10 \%$ & Voltage $+15 \%$ & $1.125 \mathrm{amps}$ & 0 amps \\
\hline Measured Input (vac) & 230.0 & 218.5 & 207.0 & 195.5 & 241.5 & 253.0 & 263.7 & 230.0 & 230.0 \\
\hline Measured Output (vdc) & 17.970 & 17.969 & 17.969 & 17.969 & 17.969 & 17.969 & 17.969 & 17.971 & 17.974 \\
\hline Output Ripple (mvac) & 8.4 & 8.7 & 8.7 & 8.6 & 8.6 & 8.7 & 8.8 & 8.7 & 8.1 \\
\hline
\end{tabular}

Condensation Conditions

Date/Time: 9/14/03 1:00 PM Full Load=1.5amps

29.7 Deg C

$91.7 \% \mathrm{RH}$

\begin{tabular}{|l|l|}
\hline $75 \%$ Load & No Load \\
\hline
\end{tabular}

Measured Input (vac) Measured Output (vdc) Output Ripple (mvac)

Comments:

\begin{tabular}{|c|c|c|c|c|c|c|c|c|}
\hline Normal Voltage & Voltage $-5 \%$ & Voltage $-10 \%$ & Voltage -15\% & Voltage $+5 \%$ & Voltage $+10 \%$ & Voltage $+15 \%$ & $1.125 \mathrm{amps}$ & 0 amps \\
\hline 8.6 & 8.8 & 8.8 & 8.5 & 8.6 & 9.0 & 8.9 & 8.8 & 8.4 \\
\hline
\end{tabular}

Reference Conditions (20 Deg C)

Date/Time: 9/15/03 9:45 AM Full Load=1.5amps 19.9 Deg C

filename $=$ CondB

8.9

8.8

\begin{tabular}{|c|c|c|c|c|c|c|c|c|c|}
\hline \multicolumn{2}{|c|}{ Date/Time: 9/15/03 9:45 AM } & \multicolumn{2}{|c|}{ Full Load=1.5amps } & \multicolumn{2}{|c|}{ 19.9 Deg C } & \multicolumn{2}{|c|}{$34.3 \% \mathrm{RH}$} & $75 \%$ Load & No Load \\
\hline & Normal Voltage & Voltage $-5 \%$ & Voltage $-10 \%$ & Voltage $-15 \%$ & Voltage $+5 \%$ & Voltage $+10 \%$ & Voltage $+15 \%$ & $1.125 \mathrm{amps}$ & 0 amps \\
\hline Measured Input (vac) & 230.0 & 218.5 & 207.0 & 195.5 & 241.5 & 253.0 & 263.7 & 230.0 & 230.0 \\
\hline Measured Output (vdc) & 17.971 & 17.971 & 17.971 & 17.971 & 17.971 & 17.970 & 17.970 & 17.972 & 17.975 \\
\hline Output Ripple (mvac) & 8.4 & 8.1 & 8.5 & 8.2 & 8.3 & 8.5 & 8.6 & 8.4 & 8.0 \\
\hline
\end{tabular}

Static Discharge Test

4kv contact discharge: With each discharge the oscilloscope displayed a "burst" waveform ( $0.7 \mathrm{~ms}$ duration, $4.6 \mathrm{vp}-\mathrm{p})$ on the $18 \mathrm{volts}$ power supply output. 8kv air discharge: With each discharge the oscilloscope displayed a "burst" waveform ( $0.8 \mathrm{~ms}$ duration, $8.4 \mathrm{vp}$-p) on the 18 volts power supply output. Comments: Applied 20 discharges (10 contact \& 10 air). The power supply continued functioning during the test and afterwards.

\begin{tabular}{|c|c|c|c|c|c|c|c|c|c|}
\hline \multicolumn{10}{|c|}{ After Static Discharge } \\
\hline \multirow[t]{2}{*}{ Date/Time: } & 9/15/03 1:30 PM & Full Load $=1.5 \mathrm{a}$ & & 21. & Deg C & 53.5 & $\% \mathrm{RH}$ & $75 \%$ Load & No Load \\
\hline & Normal Voltage & Voltage $-5 \%$ & Voltage $-10 \%$ & Voltage $-15 \%$ & Voltage $+5 \%$ & Voltage $+10 \%$ & Voltage $+15 \%$ & $1.125 \mathrm{amps}$ & 0 amps \\
\hline \multirow{3}{*}{$\begin{array}{r}\text { Measured Input (vac) } \\
\text { Measured Output (vdc) } \\
\text { Output Ripple (mvac) }\end{array}$} & 230.0 & 218.5 & 207.0 & 195.5 & 241.5 & 253.0 & 263.7 & 230.0 & 230.0 \\
\hline & 17.967 & 17.967 & 17.967 & 17.967 & 17.967 & 17.967 & 17.967 & 17.968 & 17.971 \\
\hline & 8.0 & 8.4 & 8.4 & 8.3 & 8.5 & 8.1 & 8.2 & 8.3 & 7.9 \\
\hline
\end{tabular}

Testing performed by Fred Gibson 


\section{Temperature/Humidity Testing Data - Lambda PS EWS25-18 18volts}

\begin{tabular}{|c|c|c|c|c|c|c|c|c|c|}
\hline & & & 5 Degrees & & & & & & \\
\hline Date/Time: & 9/16/03 8:30 AM & Full Load=1.5a & & & Deg C & 18.0 & $\% \mathrm{RH}$ & $75 \%$ Load & No Load \\
\hline & Normal Voltage & \begin{tabular}{|l} 
Voltage $-5 \%$ \\
\end{tabular} & Voltage $-10 \%$ & Voltage $-15 \%$ & Voltage $+5 \%$ & Voltage $+10 \%$ & Voltage $+15 \%$ & $1.125 \mathrm{amps}$ & $0 \mathrm{amps}$ \\
\hline Measured Input (vac) & 230.0 & 218.5 & 207.0 & 195.5 & 241.5 & \begin{tabular}{|l|}
253.0 \\
\end{tabular} & 263.7 & 230.0 & 230.0 \\
\hline Measured Output (vdc) & 17.968 & 17.968 & 17.968 & 17.968 & 17.968 & 17.968 & 17.968 & 17.969 & 17.973 \\
\hline Output Ripple (mvac) & 8.3 & 8.0 & 8.4 & 8.4 & 8.4 & 8.4 & 8.5 & 8.3 & 8.0 \\
\hline
\end{tabular}

45 Degrees C

\begin{tabular}{|c|c|c|c|c|c|c|c|c|c|}
\hline \multicolumn{2}{|c|}{ Date/Time: 9/17/03 8:20 AM } & \multicolumn{2}{|c|}{ Full Load=1.5amps } & \multicolumn{2}{|c|}{ 44.8 Deg C } & \multicolumn{2}{|c|}{$10.9 \% \mathrm{RH}$} & $75 \%$ Load & No Load \\
\hline & Normal Voltage & Voltage - $5 \%$ & Voltage -10\% & Voltage -15\% & Voltage $+5 \%$ & Voltage $+10 \%$ & Voltage $+15 \%$ & $1.125 \mathrm{amps}$ & 0 amps \\
\hline Measured Input (vac) & 230.0 & 218.5 & 207.0 & 195.5 & 241.5 & 253.0 & 263.7 & 230.0 & 230.0 \\
\hline Measured Output (vdc) & 17.965 & 17.965 & 17.965 & 17.965 & 17.965 & 17.965 & 17.964 & 17.966 & 17.970 \\
\hline Output Ripple (mvac) & 8.9 & 8.9 & 8.8 & 8.7 & 8.8 & 8.8 & 8.9 & 8.6 & 8.3 \\
\hline
\end{tabular}

Comments:

Reference Conditions (20 Deg C)

Date/Time: 9/17/03 3:15 PM Full Load=1.5amps

19.8 Deg C

\begin{tabular}{|c|c|c|c|c|c|c|c|c|c|}
\hline \multirow[b]{2}{*}{ - } & & 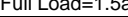 & & & & & & 7. & \\
\hline & Normal Voltage & Voltage -5\% & Voltage -10\% & Voltage -15\% & Voltage $+5 \%$ & Voltage $+10 \%$ & Voltage $+15 \%$ & $1.125 \mathrm{amps}$ & 0 amps \\
\hline \multirow{3}{*}{$\begin{array}{r}\text { Measured Input (vac) } \\
\text { Measured Output (vdc) } \\
\text { Output Ripple (mvac) }\end{array}$} & 230.0 & 218.5 & 207.0 & 195.5 & 241.5 & 253.0 & 263.7 & 230.0 & 230.0 \\
\hline & 17.962 & 17.962 & 17.962 & 17.962 & 17.963 & 17.962 & 17.962 & 17.964 & 17.967 \\
\hline & 7.9 & 7.8 & 7.8 & 7.7 & 7.8 & 7.5 & 7.7 & 7.5 & 7.2 \\
\hline
\end{tabular}
Comments:

Humidity Test (40 Deg C, 93\% RH)

\begin{tabular}{l} 
Date/Time: 9/18/03 11:00 AM \\
\cline { 2 - 12 }
\end{tabular}

Humidity Test (40 Deg C, 93\% RH)

Date/Time: 9/18/03 9:20 PM Full Load=1.5amps 40.0 Deg C

$92.1 \% \mathrm{RH}$

\begin{tabular}{|c|c|c|c|c|c|c|c|c|}
\cline { 3 - 8 } & \multicolumn{2}{c}{$45 \%$ Load } & No Load \\
\hline Normal Voltage & Voltage $-5 \%$ & Voltage $-10 \%$ & Voltage $-15 \%$ & Voltage $+5 \%$ & Voltage $+10 \%$ & Voltage $+15 \%$ & $1.125 \mathrm{amps}$ & $0 \mathrm{amps}$ \\
\hline 230.0 & 218.5 & 207.0 & 195.5 & 241.5 & 253.0 & 263.7 & 230.0 & 230.0 \\
\hline 17.970 & 17.970 & 17.970 & 17.970 & 17.970 & 17.970 & 17.969 & 17.971 & 17.975 \\
\hline 8.1 & 8.2 & 8.2 & 8.2 & 8.1 & 8.3 & 8.3 & 8.1 & 7.8 \\
\hline
\end{tabular}

Measured Input (vac) Measured Output (vdc) Output Ripple (mvac) Comments:

Humidity Test (40 Deg C, 93\% RH)

Date/Time: 9/19/03 9:20 AM Full Load=1.5amps 39.7 Deg C

\begin{tabular}{|c|c|c|c|c|c|c|c|c|c|}
\hline \multicolumn{2}{|c|}{ Date/Time: 9/19/03 9:20 AM } & \multicolumn{2}{|c|}{ Full Load=1.5amps } & \multicolumn{2}{|c|}{ 39.7 Deg C } & \multicolumn{2}{|c|}{$91.4 \% \mathrm{RH}$} & $75 \%$ Load & No Load \\
\hline & Normal Voltage & Voltage -5\% & Voltage -10\% & Voltage - $15 \%$ & Voltage $+5 \%$ & Voltage $+10 \%$ & Voltage $+15 \%$ & $1.125 \mathrm{amps}$ & $0 \mathrm{amps}$ \\
\hline Measured Input (vac) & 230.0 & 218.5 & 207.0 & 195.5 & 241.5 & 253.0 & 263.7 & 230.0 & 230.0 \\
\hline Measured Output (vdc) & 17.970 & 17.970 & 17.970 & 17.970 & 17.970 & 17.970 & 17.970 & 17.971 & 17.975 \\
\hline Output Ripple (mvac) & 8.1 & 8.1 & 8.0 & 8.0 & 8.2 & 8.1 & 8.3 & 8.0 & 7.4 \\
\hline
\end{tabular}

Reference Conditions (20 Deg C)

Date/Time: 9/19/03 8:20 PM Full Load=1.5amps 19.9 Deg C

\begin{tabular}{|c|c|c|c|c|c|c|c|c|c|}
\hline & & & & & \multirow[b]{2}{*}{$1.125 \mathrm{amps}$} & \multirow[b]{2}{*}{0 amps } \\
\hline & Normal Voltage & Voltage -5\% & Voltage -10\% & Voltage $-15 \%$ & Voltage $+5 \%$ & Voltage $+10 \%$ & Voltage $+15 \%$ & & \\
\hline Measured Input (vac) & 230.0 & 218.5 & 207.0 & 195.5 & 241.5 & 253.0 & 263.7 & 230.0 & 230.0 \\
\hline Measured Output (vdc) & 17.964 & 17.964 & 17.964 & 17.964 & 17.964 & 17.964 & 17.964 & 17.966 & 17.969 \\
\hline Output Ripple (mvac) & 6.9 & 6.9 & 7.0 & 6.9 & 7.0 & 7.0 & 7.0 & 6.8 & 6.2 \\
\hline
\end{tabular}


RF Emissions

Ambient and Lambda Power Supply

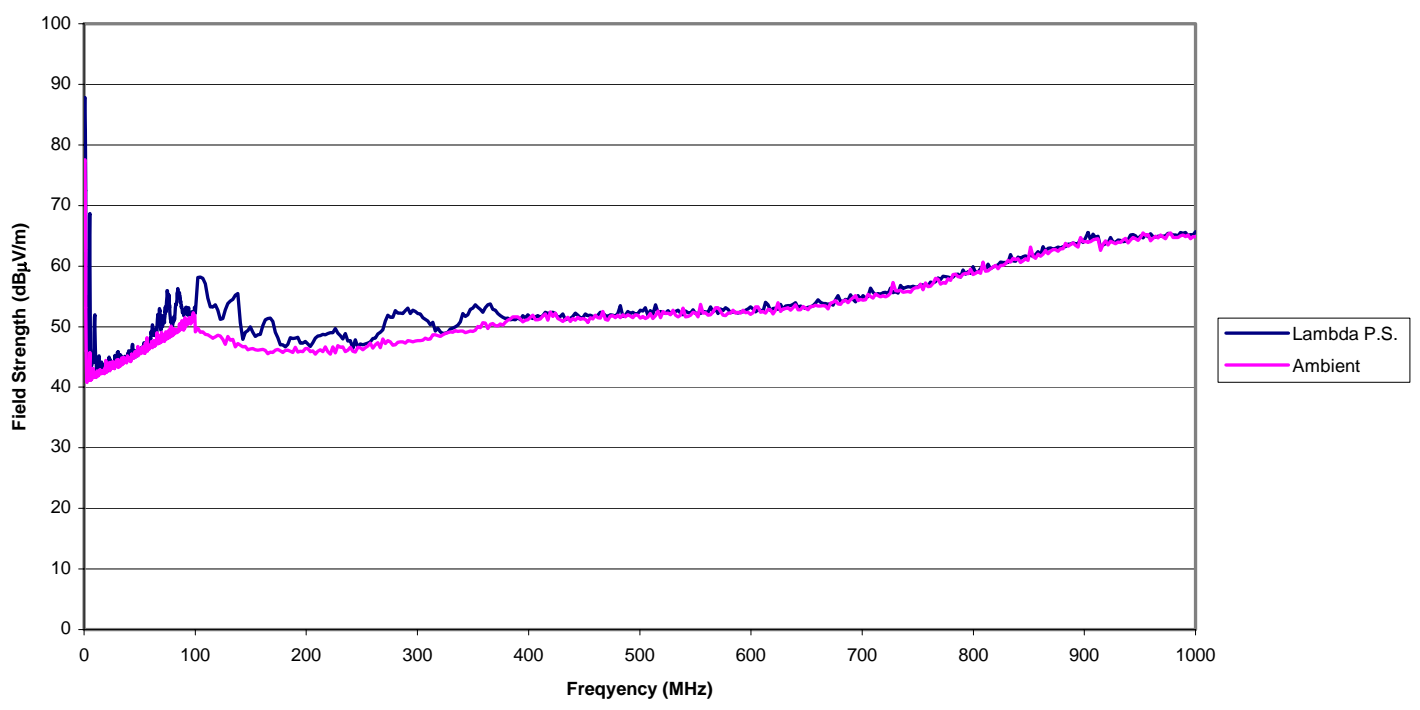

D - 8 


\section{Testing Data - Lambda PS EWS25-18 18volts}

Electrical fast transient test (100 $\mathrm{kHz}$ ring wave)

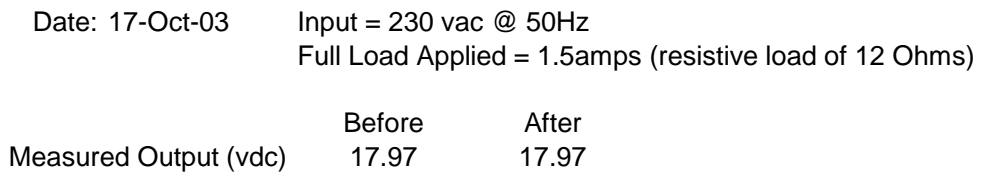

A total of 10 ring wave bursts (each $2 \mathrm{kv}$ ) were applied to the power supply at intervals of 1 minute. No functional abnormalities were noticed during nor after the test.

\section{Surge test (1.2/50 us - 8/20 us combination wave)}

Date: $17-O c t-03 \quad$ Input $=230$ vac @ $50 \mathrm{~Hz}$

Full Load Applied = 1.5amps (resistive load of $12 \mathrm{Ohms}$ )

$\begin{array}{ccc} & \text { Before } & \text { After } \\ \text { Measured Output (vdc) } & 17.97 & 17.97\end{array}$

A total of 10 combination waves (each $2 \mathrm{kv}$ ) were applied to the power supply at intervals of 1 minute. No functional abnormalities were noticed during nor after the test.

\section{Testing Data - Lambda PS EWS25-18 18volts}

\section{Radiated Radio Frequency Field}

Frequency Range 80 to $1000 \mathrm{MHz}$

Frequency step size 1\%

Dwell time 5 seconds

Date: 16-Oct-03

Ambient: 22.1 Deg C, 42.2\% RH, 29.28 in HG

Input = 230 vac @ $90 \mathrm{~Hz}$

Full Load Applied = 1.5amps (resistive load of $12 \mathrm{Ohms}$ )

$\begin{array}{rc}\text { Measured Output (vdc) } & 17.96 \\ \text { Measured Ripple (mv ac) } & 2.27\end{array}$

\section{With No RF Applied}

\section{Observations:}

Between 249 and $349 \mathrm{MHz}$ the output voltage dipped to 17.85 and $17.90 \mathrm{vdc}$ Between 339 and $341 \mathrm{MHz}$ the ripple voltage went up to $10 \mathrm{mv}$.

\section{Comments:}

Both of these perturbations were well within the specifications of the power supply. No susceptibilities were found.

Testing performed by: Fred Gibson 


\section{Testing Data - Lambda PS EWS25-18 18volts Conducted Radio Frequency}

Frequency Range 0.15 to $80 \mathrm{MHz}$ Frequency step size $1 \%$

Dwell time 5 seconds
Injected Signal Strength $33 \mathrm{dbm}$ into CDN Injected Signal Strength $26 \mathrm{dbm}$ into EUT Modulation AM $1 \mathrm{kHz} 80 \%$

Date: $16-$ Oct-03

Ambient: 22.1 Deg C, $42.2 \% \mathrm{RH}, 29.28$ in HG

Input = 230 vac @ 50Hz

Full Load Applied = 1.5amps (resistive load of 12 Ohms)

With No RF Applied

$\begin{array}{rc}\text { Measured Output (vdc) } & 17.96 \\ \text { Measured Ripple (mv ac) } & 0.8\end{array}$

Observations:

No variation of output voltage nor ac ripple levels were observed.

Comments:

No susceptibilities were found.

Testing performed by: Fred Gibson \& Roberto Jean-Pierre

D - 10 


\section{Testing Data - Lambda PS EWS25-18 18volts}

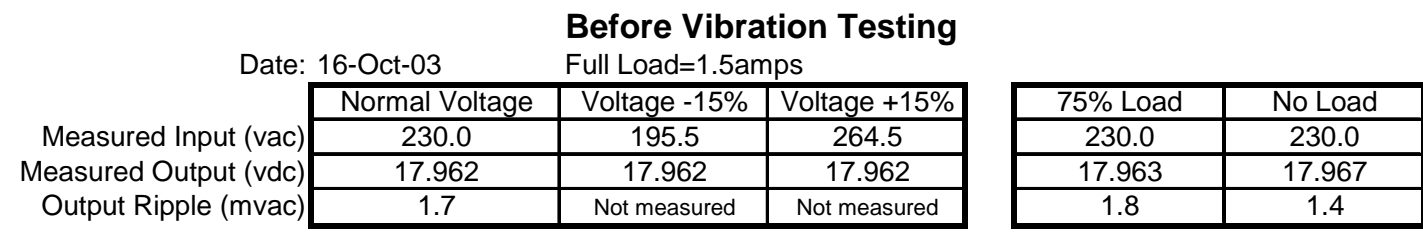

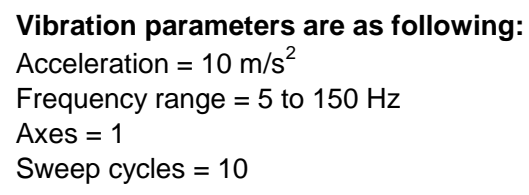

\begin{tabular}{|c|c|c|c|c|c|}
\hline & After & /ibration / Be & fore Shock T & & \\
\hline Date: & L6-Oct-03 & Full Load=1.5an & & & \\
\hline & Normal Voltage & Voltage $-15 \%$ & Voltage $+15 \%$ & $75 \%$ Load & No Load \\
\hline Measured Input (vac) & 230.0 & 195.5 & 264.5 & 230.0 & 230.0 \\
\hline Measured Output (vdc) & 17.958 & 17.958 & 17.958 & 17.959 & 17.963 \\
\hline Output Ripple (mvac) & 1.7 & Not measured & Not measured & 1.7 & 1.3 \\
\hline
\end{tabular}

Shock parameters are as following:

Peak acceleration $=150 \mathrm{~m} / \mathrm{s}^{2}$

Duration $=6 \mathrm{~ms}$

Number of shocks $=100$ (in one direction only)

After Shock Testing

Date: 16-Oct-03 Full Load=1.5amps

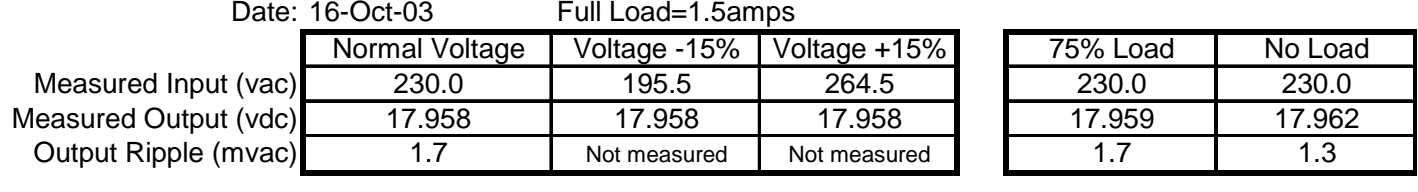

Observations:

No anomalies were observed during or after each test.

No susceptibilities in the direction that was tested.

Testing was performed by Fred Gibson 


\section{TEST RESULTS \\ FOR \\ UNINTERRUPTIBLE POWER SUPPLY \\ APC SU22-XL}

D -12 
Testing Data - APC UPS SU2200XL

\begin{tabular}{|c|c|c|c|c|c|c|c|c|c|}
\hline \multirow[t]{5}{*}{ Date/Time: } & 9/9/03 2:25 PM & \multirow{2}{*}{\multicolumn{4}{|c|}{$\begin{array}{l}\text { Nominal Environmental Conditions (20C) } \\
\text { On Mains }\end{array}$}} & \multicolumn{2}{|c|}{ 22.4 Deg C } & \multicolumn{2}{|c|}{$53.0 \% \mathrm{RH}$} \\
\hline & & & & & & \multirow[b]{2}{*}{ Resp. time } & \multicolumn{3}{|c|}{ Output after Transfer } \\
\hline & Voltage & Current & Power & Volts at Transfer Dn & Volts at Transfer Up & & Distortion & Voltage & Frequency \\
\hline & (vac) & (A) & (Watts) & \begin{tabular}{|c|} 
(vac) \\
\end{tabular} & (vac) & (ms) & $(\%)$ & (vac) & $(\mathrm{Hz})$ \\
\hline & 230.0 & 5.01 & 1152 & 195.6 & & $<2$ & 1.4 & 216.6 & 50.03 \\
\hline
\end{tabular}

\begin{tabular}{|c|c|c|c|c|c|c|c|c|c|}
\hline \multirow[t]{5}{*}{ Date/Time: } & \multicolumn{5}{|c|}{ Temperature (5 Deg C) } & \multicolumn{2}{|c|}{ 4.8 Deg C } & \multicolumn{2}{|c|}{$17.6 \% \mathrm{RH}$} \\
\hline & \multicolumn{5}{|c|}{ On Mains } & \multirow[b]{2}{*}{ Resp. time } & \multicolumn{3}{|c|}{ Output after Transfer } \\
\hline & Voltage & Current & Power & Volts at Transfer Dn & Volts at Transfer Up & & Distortion & Voltage & Frequency \\
\hline & (vac) & (A) & (Watts) & (vac) & (vac) & $(\mathrm{ms})$ & $(\%)$ & (vac) & $(\mathrm{Hz})$ \\
\hline & 230.1 & 5.02 & 1155 & 197.7 & 255.5 & $<2$ & 1.4 & 216.6 & 50.03 \\
\hline
\end{tabular}

Date/Time: $\quad$ 9/11/03 9:30 AM $\quad$ Temperature (45 Deg C)

\begin{tabular}{|c|c|c|c|c|c|c|c|c|}
\hline \multicolumn{5}{|c|}{ On Mains } & \multirow[b]{2}{*}{ Resp. time } & \multicolumn{3}{|c|}{ Output after Transfer } \\
\hline Voltage & Current & Power & Volts at Transfer Dn & Volts at Transfer Up & & Distortion & Voltage & Frequency \\
\hline (vac) & $(A)$ & (Watts) & \begin{tabular}{c|} 
(vac) \\
\end{tabular} & (vac) & (ms) & $(\%)$ & (vac) & $(\mathrm{Hz})$ \\
\hline 230.0 & 5.01 & 1152 & 195.8 & 253.6 & $<2$ & 1.3 & 216.6 & 50.03 \\
\hline
\end{tabular}

\begin{tabular}{|c|c|c|c|c|c|c|c|c|c|}
\hline \multirow[t]{5}{*}{ Date/Time: } & \multirow{2}{*}{\multicolumn{5}{|c|}{$\frac{\text { Ref. Temperature (20 Deg C) }}{\text { On Mains }}$}} & \multicolumn{2}{|c|}{ 19.9 Deg C } & \multicolumn{2}{|c|}{$11.7 \% \mathrm{RH}$} \\
\hline & & & & & & & Outp & $t$ after Tra & nnsfer \\
\hline & Voltage & Current & Power & Volts at Transfer Dn & \begin{tabular}{|l|l} 
Volts at Transfer Up \\
\end{tabular} & Resp. time & Distortion & Voltage & Frequency \\
\hline & (vac) & (A) & (Watts) & (vac) & \begin{tabular}{|c|} 
(vac) \\
\end{tabular} & (ms) & (\%) & (vac) & $(\mathrm{Hz})$ \\
\hline & 230.0 & 5.02 & 1155 & 197.0 & 254.4 & $<2$ & 1.4 & 217.9 & 50.03 \\
\hline
\end{tabular}

Electrical Discharge Test

\begin{tabular}{|c|c|c|c|c|c|c|c|c|c|}
\hline \multicolumn{2}{|c|}{ 4kv Contact Discharge Test: } & \multicolumn{8}{|c|}{$\begin{array}{l}\text { Test was performed while UPS was on the mains. } 10 \text { discharges were applied at various places on its } \\
\text { panel and its sides. } 10 \text { more discharges were applied as above but with UPS in inverter mode.. } \\
\text { No anomalies were observed. }\end{array}$} \\
\hline \multicolumn{2}{|c|}{ 8kv Air Discharge Test: } & \multicolumn{8}{|c|}{$\begin{array}{l}\text { Test was performed while UPS was on the mains. } 10 \text { discharges were applied near various places on its } \\
\text { panel and its sides. } 10 \text { more discharges were applied as above but with UPS in inverter mode.. } \\
\text { No anomalies were observed. }\end{array}$} \\
\hline \multirow[t]{5}{*}{ Date/Time: } & 9/12/03 10:22 AM & \multicolumn{4}{|c|}{ After Electrostatic Discharge } & \multicolumn{2}{|c|}{21.8 Deg C } & \multicolumn{2}{|c|}{$49.8 \% \mathrm{RH}$} \\
\hline & \multicolumn{5}{|c|}{ On Mains } & \multicolumn{4}{|c|}{\begin{tabular}{|c|c|} 
& Output after Transfer \\
\end{tabular}} \\
\hline & Voltage & Current & Power & Volts at Transfer Dn & Volts at Transfer Up & Resp. time & Distortion & Voltage & Frequency \\
\hline & (vac) & $(\mathrm{A})$ & (Watts) & (vac) & (vac) & (ms) & $(\%)$ & (vac) & $(\mathrm{Hz})$ \\
\hline & 230.0 & 5.01 & 1152 & 196.6 & 254.3 & $<2$ & 1.5 & 218.0 & 50.03 \\
\hline
\end{tabular}




\section{TEST RESULTS \\ FOR \\ UNINTERRUPTIBLE POWER SUPPLY \\ TRACE SW3048J}

D -14 


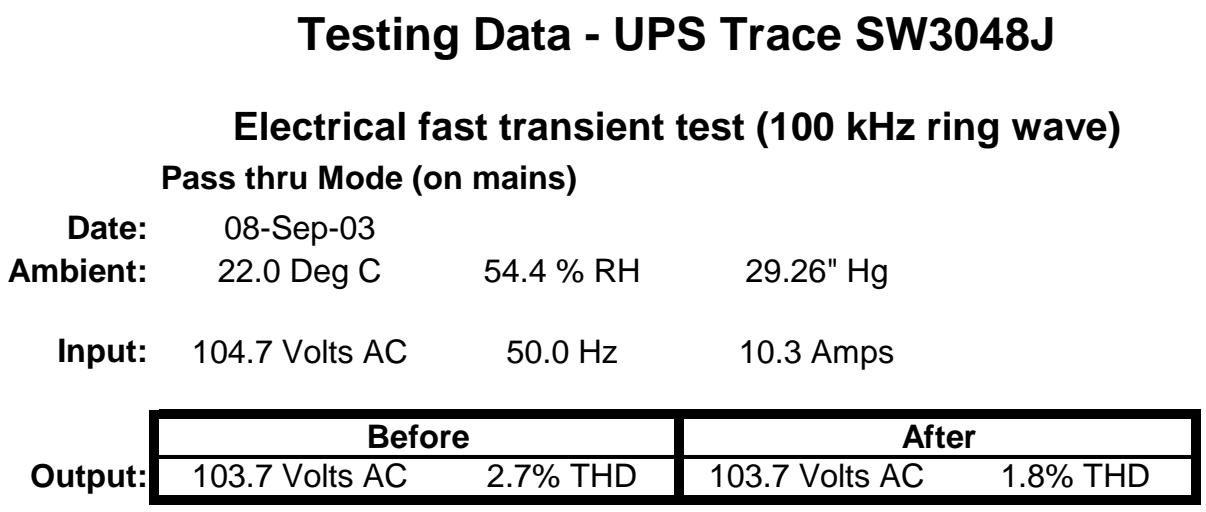

Comments: A total of 10 ring wave bursts (each $2 \mathrm{kv}$ ) were applied to the UPS at intervals intervals of 1 minute.

No functional abnormalities were noticed during nor after the test.

\section{Surge test (1.2/50 us - 8/20 us combination wave) \\ Pass thru Mode (on mains)}

Input: $\quad 104.7$ Volts AC $\quad 50.0 \mathrm{~Hz} \quad 10.3 \mathrm{Amps}$

\begin{tabular}{|c|c|c|c|}
\hline & Bef & & After \\
\hline Output: & 103.7 Volts AC & $1.9 \%$ THD & Failure see comments \\
\hline
\end{tabular}

Comments: After applying the first surge (4kv) the UPS switched to battery (inverter mode). Then after 20 seconds it switched back to mains. Functionally it looked good. After applying the second surge the UPS switched to battery again, but then started oscillating between battery and mains. The output voltage started increasing and the unit was shut down after the output voltage reached 120 volts AC.

Test was performed by: Fred Gibson 
Testing Data - Trace UPS SW3048J

Date/Time: 9/5/03 5:00 PM Nominal Environmental Conditions (20 Deg C)

\begin{tabular}{|c|c|c|c|c|c|c|c|c|}
\hline \multicolumn{5}{|c|}{ Nominal Environmental Conditions (20 Deg C) } & \multirow[b]{3}{*}{ Resp. time } & \multicolumn{3}{|c|}{ 20.1 Deg C } \\
\hline & & Mains & & & & & ut after T & fer \\
\hline Voltage & Current & Power & Volts at Transfer Dn & Volts at Transfer Up & & Distortion & Voltage & Frequency \\
\hline (vac) & (A) & (Watts) & (vac) & (vac) & (ms) & (\%) & (vac) & $(\mathrm{Hz})$ \\
\hline 105.2 & 13.7 & 1441 & 93.8 & 116.1 & $<2$ & 2.5 & 104.0 & 50.03 \\
\hline
\end{tabular}

\begin{tabular}{|c|c|c|c|c|c|c|c|c|c|}
\hline \multirow[t]{5}{*}{ Date/Time: } & \multicolumn{6}{|c|}{ Condensation Conditions } & \multicolumn{2}{|c|}{$29.9 \operatorname{Deg} C$} & $91.8 \%$ \\
\hline & \multicolumn{5}{|c|}{ Mains Input } & & \multicolumn{3}{|c|}{ Output after Transfer } \\
\hline & Voltage & Current & Power & Volts at Transfer Dn & Volts at Transfer Up & Resp. time & Distortion & Voltage & Frequency \\
\hline & (vac) & (A) & (Watts) & (vac) & (vac) & (ms) & $(\%)$ & (vac) & $(\mathrm{Hz})$ \\
\hline & 105.5 & 13.8 & 1456 & 93.9 & 117.0 & $<2$ & 2.4 & 103.1 & 50.03 \\
\hline & & & & & & filename $=\mathrm{Co}$ & $\mathrm{ddA} \ldots$ & & \\
\hline \multirow[t]{6}{*}{ Date/Time: } & \multicolumn{5}{|c|}{ Condensation Conditions } & & \multicolumn{2}{|c|}{29.9 Deg C } & $92.1 \%$ \\
\hline & \multicolumn{5}{|c|}{ Mains Input } & & \multicolumn{3}{|c|}{ Output after Transfer } \\
\hline & Voltage & Current & Power & Volts at Transfer Dn & Volts at Transfer Up & Resp. time & Distortion & Voltage & Frequency \\
\hline & (vac) & (A) & (Watts) & (vac) & (vac) & (ms) & $(\%)$ & (vac) & $(\mathrm{Hz})$ \\
\hline & 105.3 & 13.7 & 1443 & 93.5 & 118.1 & $<2$ & 2.4 & 103.1 & 50.03 \\
\hline & & & & & & filename $=\mathrm{Co}$ & $\mathrm{ddB} \ldots$ & & \\
\hline \multirow[t]{5}{*}{ Date/Time: } & \multirow{2}{*}{\multicolumn{5}{|c|}{ Reference Conditions (20 Deg C) }} & & \multicolumn{2}{|c|}{ 19.9 Deg C } & $41.3 \%$ \\
\hline & \multicolumn{4}{|c|}{ Mains Input } & & & \multicolumn{3}{|c|}{ Output after Transfer } \\
\hline & Voltage & Current & Power & Volts at Transfer Dn & Volts at Transfer Up & Resp. time & Distortion & Voltage & Frequency \\
\hline & (vac) & (A) & (Watts) & (vac) & (vac) & $(\mathrm{ms})$ & $(\%)$ & (vac) & $(\mathrm{Hz})$ \\
\hline & 105.9 & 13.8 & 1461 & 94.0 & 118.7 & $<2$ & 2.4 & 103.7 & 50.03 \\
\hline
\end{tabular}

\section{Electrical Discharge Test}

See reference condition above for pre-discharge electrical data.

$$
\begin{array}{lll}
22.0 \mathrm{Deg} \mathrm{C} & 54.4 \% \mathrm{RH} & 29.26 \mathrm{in} \mathrm{Hg}
\end{array}
$$

4kv Contact Discharge Test: Test was performed while UPS was on the mains. 10 discharges were applied at various places on its panel and its sides. 10 more discharges were applied as above but with UPS in inverter mode..

No anomalies were observed.

8kv Air Discharge Test: Test was performed while UPS was on the mains. 10 discharges were applied near various places on its panel and its sides. 10 more discharges were applied as above but with UPS in inverter mode..

No anomalies were observed.

Date/Time: 9/8/03 1:45 PM

After Static Discharge: No anomalies on mains and on battery

\begin{tabular}{|c|c|c|c|c|c|c|c|c|}
\hline \multicolumn{5}{|c|}{ Mains Input } & \multirow[b]{2}{*}{ Resp. time } & \multicolumn{3}{|c|}{ Output after Transfer } \\
\hline Voltage & Current & Power & Volts at Transfer Dn & Volts at Transfer Up & & Distortion & Voltage & Frequency \\
\hline (vac) & (A) & (Watts) & (vac) & (vac) & (ms) & (\%) & (vac) & $(\mathrm{Hz})$ \\
\hline 105.2 & 13.6 & 1431 & 93.6 & 117.7 & $<2$ & 2.9 & 102.1 & 50.03 \\
\hline
\end{tabular}




\section{TEST RESULTS \\ FOR \\ UNINTERRUPTIBLE POWER SUPPLY \\ TRACE DR1512E}

D - 17 
Testing Data - Trace UPS DR1512E

\begin{tabular}{|c|c|c|c|c|c|c|c|}
\hline \multicolumn{6}{|c|}{ Nominal Environmental Conditions (20C) } & & \\
\hline & & & & & \multicolumn{3}{|c|}{ Output after Transfer } \\
\hline Voltage & Current & Power & Volts at Transfer & Resp. time & Distortion & Voltage & Frequency \\
\hline (vac) & (A) & (Watts) & (vac) & $(\mathrm{ms})$ & $(\%)$ & (vac) & $(\mathrm{Hz})$ \\
\hline 230.0 & 4.02 & 925 & 199.9 & $<2$ & 27.2 & 219.2 & 50.00 \\
\hline
\end{tabular}

\begin{tabular}{|c|c|c|c|c|c|c|c|}
\hline \multicolumn{2}{|l|}{ 8/29/03 10:00 AM } & \multicolumn{3}{|c|}{ Temperature (5 Deg C) } & \multicolumn{2}{|c|}{ 4.9 Deg C } & $19.0 \% \mathrm{RH}$ \\
\hline \multicolumn{4}{|c|}{ Mains Input } & & \multicolumn{3}{|c|}{ Output after Transfer } \\
\hline Voltage & Current & Power & Volts at Transfer & Resp. time & Distortion & Voltage & Frequency \\
\hline (vac) & (A) & (Watts) & (vac) & (ms) & $(\%)$ & (vac) & $(\mathrm{Hz})$ \\
\hline 230.0 & 4.04 & 929 & 202.0 & $<2$ & 29.3 & 231.6 & 50.00 \\
\hline \multicolumn{2}{|l|}{ 8/30/03 7:00 AM } & \multicolumn{3}{|c|}{ Temperature (45 Deg C) } & \multicolumn{2}{|c|}{ 44.8 Deg C } & $5.0 \% \mathrm{RH}$ \\
\hline \multicolumn{4}{|c|}{ Mains Input } & & \multicolumn{3}{|c|}{ Output after Transfer } \\
\hline Voltage & Current & Power & Volts at Transfer & Resp. time & Distortion & Voltage & Frequency \\
\hline (vac) & (A) & (Watts) & (vac) & (ms) & $(\%)$ & (vac) & $(\mathrm{Hz})$ \\
\hline 230.0 & 4.04 & 929 & 199.4 & $<2$ & 28.7 & 228.3 & 50.00 \\
\hline \multicolumn{5}{|c|}{ Temperature (20 Deg C) } & \multicolumn{3}{|c|}{ 19.8 Deg C } \\
\hline \multicolumn{4}{|c|}{ Mains Input } & & \multicolumn{3}{|c|}{ Output after Transfer } \\
\hline Voltage & Current & Power & Volts at Transfer & Resp. time & Distortion & Voltage & Frequency \\
\hline (vac) & (A) & (Watts) & (vac) & $(\mathrm{ms})$ & $(\%)$ & (vac) & $(\mathrm{Hz})$ \\
\hline 229.2 & 4.02 & 921 & 200.9 & $<2$ & 28.2 & 229.3 & 50.00 \\
\hline
\end{tabular}




\begin{tabular}{|c|c|c|c|c|c|c|c|}
\hline \multicolumn{4}{|c|}{ Mains Input } & \multirow{3}{*}{} & \multicolumn{3}{c|}{ Output after Transfer } \\
\cline { 6 - 9 } & Current & Power & Volts at Transfer & Resp. time & Distortion & Voltage & Frequency \\
\hline Voltage & (A) & (Watts) & (vac) & $(\mathrm{ms})$ & $(\%)$ & $(\mathrm{vac})$ & $(\mathrm{Hz})$ \\
\hline 230.3 & 4.03 & 928 & 199.9 & $<2$ & 29.0 & 227.9 & 50.00 \\
\hline
\end{tabular}

File name $=$ HumA...

8/31/03 8:00 PM Humidity (40 Deg C, 93\%RH)

40.0 Deg C

$92.5 \% \mathrm{RH}$

\begin{tabular}{|c|c|c|c|c|c|c|c|}
\hline \multicolumn{4}{|c|}{ Mains Input } & \multirow{3}{*}{ Output after Transfer } \\
\cline { 7 - 9 } & Current & Power & Volts at Transfer & Resp. time & Distortion & Voltage & Frequency \\
\hline Voltage & (vac) & $($ Watts $)$ & $(\mathrm{vac})$ & $(\mathrm{ms})$ & $(\%)$ & $(\mathrm{vac})$ & $(\mathrm{Hz})$ \\
\hline 230.2 & 4.02 & 925 & 199.9 & $<2$ & 28.8 & 227.9 & 50.00 \\
\hline
\end{tabular}

File name $=$ HumB...

\begin{tabular}{|c|c|c|c|c|c|c|c|}
\hline 9/1/03 8:0 & \multicolumn{4}{|c|}{ Humidity (40 Deg C, 93\%RH) } & \multicolumn{2}{|c|}{ 40.3 Deg C } & 93.3 \\
\hline & \multicolumn{2}{|c|}{ Mains Input } & & \multirow[b]{2}{*}{ Resp. time } & & it after T & \\
\hline Voltage & Current & Power & Volts at Transfer & & Distortion & Voltage & Frequency \\
\hline (vac) & $(\mathrm{A})$ & (Watts) & (vac) & (ms) & $(\%)$ & (vac) & $(\mathrm{Hz})$ \\
\hline 230.3 & 4.02 & 926 & 199.9 & $<2$ & 29.1 & 229.2 & 50.00 \\
\hline
\end{tabular}

File name $=$ HumC.

9/1/03 8:00 PM

Humidity (40 Deg C, 93\%RH)

39.9 Deg C

$92.1 \% \mathrm{RH}$

\begin{tabular}{|c|c|c|c|c|c|c|c|}
\hline \multicolumn{4}{|c|}{ Mains Input } & \multirow[b]{2}{*}{ Resp. time } & \multicolumn{3}{|c|}{ Output after Transfer } \\
\hline Voltage & Current & Power & Volts at Transfer & & Distortion & Voltage & Frequency \\
\hline (vac) & (A) & (Watts) & (vac) & (ms) & $(\%)$ & (vac) & $(\mathrm{Hz})$ \\
\hline 230.3 & 4.02 & 926 & 199.8 & $<2$ & 29.0 & 229.2 & 50.00 \\
\hline
\end{tabular}

File name $=$ HumD..

9/2/03 8:00 AM Humidity (40 Deg C, 93\%RH)

40.1 Deg C

$92.5 \% \mathrm{RH}$

\begin{tabular}{|c|c|c|c|c|c|c|c|}
\hline \multicolumn{4}{|c|}{ Mains Input } & \multirow[b]{2}{*}{ Resp. time } & \multicolumn{3}{|c|}{ Output after Transfer } \\
\hline Voltage & Current & Power & Volts at Transfer & & Distortion & Voltage & Frequency \\
\hline (vac) & (A) & (Watts) & (vac) & (ms) & $(\%)$ & (vac) & $(\mathrm{Hz})$ \\
\hline 230.3 & 4.03 & 928 & 199.8 & $<2$ & 28.1 & 227.9 & 50.00 \\
\hline
\end{tabular}

File name $=$ HumE...

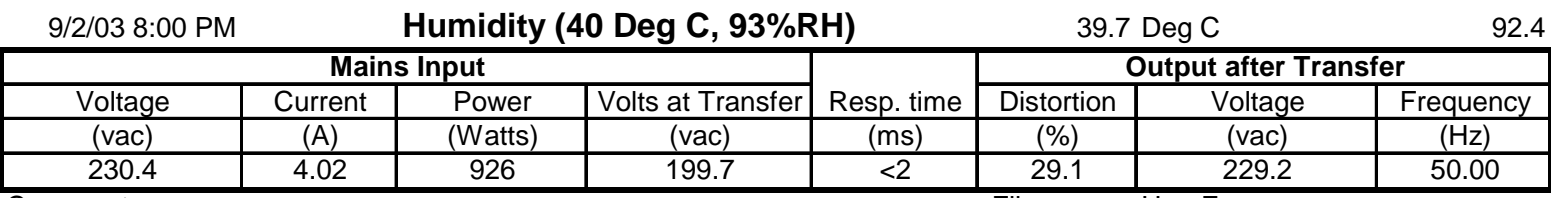

Comments:

File name $=$ HumF..

After a 70\% Dip, the UPS shutdown (no lights). A manual restart was required to restart the UPS.

Several more $70 \%$ dips were done with good responses, no UPS shutdowns.

\begin{tabular}{|c|c|c|c|c|c|c|c|}
\hline 9/3/03 8:0 & \multicolumn{4}{|c|}{ Humidity (40 Deg C, 93\%RH) } & \multicolumn{2}{|c|}{ 39.8 Deg C } & 91.7 \\
\hline & \multicolumn{3}{|c|}{ Mains Input } & \multirow[b]{2}{*}{ Resp. time } & \multicolumn{3}{|c|}{ Output after Transfer } \\
\hline Voltage & Current & Power & Volts at Transfer & & Distortion & Voltage & Frequency \\
\hline (vac) & $(\mathrm{A})$ & (Watts) & (vac) & (ms) & $(\%)$ & (vac) & $(\mathrm{Hz})$ \\
\hline 230.3 & 4.03 & 928 & 199.9 & $<2$ & 29.1 & 230.4 & 50.00 \\
\hline
\end{tabular}

File name $=$ HumG...

9/3/03 8:00 PM

Humidity (40 Deg C, 93\%RH)

\begin{tabular}{|c|c|c|c|c|c|c|c|}
\hline \multicolumn{4}{|c|}{ Mains Input } & \multirow[b]{2}{*}{ Resp. time } & \multicolumn{3}{|c|}{ Output after Transfer } \\
\hline Voltage & Current & Power & Volts at Transfer & & Distortion & Voltage & Frequency \\
\hline (vac) & $(\mathrm{A})$ & (Watts) & (vac) & (ms) & $(\%)$ & (vac) & $(\mathrm{Hz})$ \\
\hline 230.3 & 4.03 & 928 & 199.8 & $<2$ & 29.4 & 230.4 & 50.00 \\
\hline
\end{tabular}

File name $=\mathrm{HumH} \ldots$

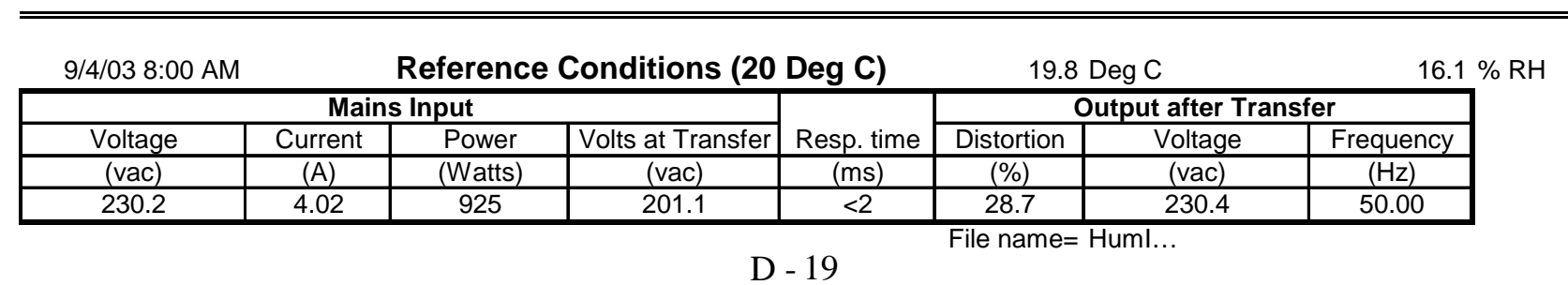




\section{Electrical Discharge Test}

See reference condition above for pre-discharge electrical data.
21.2 Deg C
29.1" $\mathrm{Hg}$
$59.6 \% \mathrm{RH}$

t Discharge Test: Test was performed while UPS was on the mains. 10 discharges were applied at various places on its panel and its sides. 10 more discharges were applied as above but with UPS in inverter mode..

No anomalies were observed.

:harge Test: $\quad$ Test was performed while UPS was on the mains. 10 discharges were applied near various places on its panel and its sides. 10 more discharges were applied as above but with UPS in inverter mode..

An air discharge was applied near the "battery low/high" light which shutdown the UPS while it was in the inverter mode. The on/off button was pressed restarting the UPS.

9/4/03 11:00 AM After Electrical Discharge Test

21.2 Deg C

29.1" Hg

$59.6 \% \mathrm{RH}$ Mains Input 


\section{Testing Data - Trace UPS DR1512E}

Date: 15 -Oct-03 Input: 230 Volts $A C$ Output: 229 Volts AC
Before Vibration Testing

\author{
Pass thru mode (on mains)
}

$50 \mathrm{~Hz}$

$50 \mathrm{~Hz}$
$1 \mathrm{kw}$ load

4.06 Amps $\quad 1.0 \%$ THD

Vibration parameters are as following:

Acceleration $=10 \mathrm{~m} / \mathrm{s}^{2}$

Frequency range $=5$ to $150 \mathrm{~Hz}$

Direction $=1$

Sweep cycles $=10$

Date: $15-O c t-03$

Output: 230.6 Volts AC

After Vibration / Before Shock Testing

Inverter mode (on battery)

$50 \mathrm{~Hz} \quad 1 \mathrm{kw}$ load

$28.0 \%$ THD

\begin{tabular}{|c|c|c|c|}
\hline \multicolumn{4}{|c|}{$\begin{array}{l}\text { Shock parameters are as following: } \\
\text { Peak acceleration }=150 \mathrm{~m} / \mathrm{s}^{2} \\
\text { Duration }=8 \mathrm{~ms} \\
\text { Number of shocks }=100 \text { (in one direction only) }\end{array}$} \\
\hline \multicolumn{4}{|c|}{ After Shock Testing } \\
\hline $\begin{array}{l}\text { Date: } 16-\text { Oct-03 } \\
\text { Input: } 229 \text { Volts AC }\end{array}$ & $\begin{array}{l}\text { Pass t } \\
50 \mathrm{~Hz}\end{array}$ & le (on main & \\
\hline \multirow[t]{2}{*}{ Output: 229 Volts AC } & $50 \mathrm{~Hz}$ & $1 \mathrm{kw}$ load & $0.9 \%$ THD \\
\hline & Invert & (on battery & \\
\hline Output: 229 Volts AC & $50 \mathrm{~Hz}$ & $1 \mathrm{kw}$ load & $27.8 \%$ THD \\
\hline
\end{tabular}

Observations:

No anomalies were observed during or after each test.

No susceptibilities in the direction that was tested.

Testing was performed by Fred Gibson \& Roberto Jean-Pierre 


\section{Testing Data - Trace UPS DR1512E}

\section{Conducted Radio Frequency}

Frequency Range 0.15 to $80 \mathrm{MHz}$

Frequency step size $1 \%$

Dwell time 5 seconds
Injected Signal Strength $33 \mathrm{dbm}$ into CDN Injected Signal Strength $26 \mathrm{dbm}$ into EUT

Modulation AM $1 \mathrm{kHz} 80 \%$

Date: $14-O c t-03$

Ambient: 22.6 Deg C, 58.2\% RH, 29.75 in HG

Measured Input $=230$ vac $@ 50 \mathrm{~Hz}$

Pass thru mode (UPS on mains) - no RF applied

Measured Output $w / 1 \mathrm{kw}$ Load $=229$ vac, $4.02 \mathrm{amps}, 1.0 \%$ THD

Pass thru mode (UPS on mains) - RF applied

Observations: Between 60 and $70 \mathrm{MHz}$ THD went to 4 - 7\%

Between 76 and $80 \mathrm{MHz}$ UPS switched to inverter mode

Measured Input = 0 vac (input voltage not connected)

Inverter mode (UPS on battery) - no RF applied

Measured Output w/500w Load = $225 \mathrm{vac}, 1.96 \mathrm{amps}, 28.3 \% \mathrm{THD}, 50 \mathrm{~Hz}$

Inverter mode (UPS on battery) - RF applied

Observations: Between 65 and $80 \mathrm{MHz}$ the over temp LED came on and the output voltage went up. When the output voltage reached $257 \mathrm{vac}$, the UPS shut down.

Testing performed by: Fred Gibson \& Roberto Jean-Pierre 


\section{Testing Data - Trace UPS DR1512E}

\section{Radiated Radio Frequency}

Frequency Range 80 to $1000 \mathrm{MHz}$

Frequency step size $1 \%$

Dwell time 5 seconds
Field Strength $10 \mathrm{v} / \mathrm{m} \& 20 \mathrm{v} / \mathrm{m}$

Modulation AM $1 \mathrm{kHz} 80 \%$

Date: $13-O c t-03$

Ambient: 22.5 Deg C, 58.2\% RH, 29.18 in HG

Measured Input = 230 vac @ $50 \mathrm{~Hz}$

Pass thru mode (UPS on mains) - no RF applied

Measured Output w/1kw Load = 228.9 vac, 4.07 amps, $1.1 \%$ THD

Pass thru mode (UPS on mains) - RF applied (both $10 \& 20 \mathrm{v} / \mathrm{m}$ )

Observations: Susceptibilies found in 107 to $122 \mathrm{MHz}$ and 149 to $151 \mathrm{MHz}$ ranges

as outlined below:

@ $107 \mathrm{MHz}$ UPS switched to inverter mode

@ $111 \mathrm{MHz}$ Overload LED came on

@ $114 \mathrm{MHz}$ all panel LED's went off

@ $117 \mathrm{MHz}$ Overload LED started flashing

@ $122 \mathrm{MHz}$ UPS returns to normal (on mains)

107 to $122 \mathrm{MHz}$ the output voltage reached as high as 238 vac

149 to $151 \mathrm{MHz}$ the Battery High LED was flashing

Measured Input $=0$ vac $($ input voltage not connected $)$

Inverter mode (UPS on battery) - no RF applied

Measured Output w/1kw Load = $230 \mathrm{vac}, 28.2 \% \mathrm{THD}, 50 \mathrm{~Hz}$

Inverter mode (UPS on battery) - RF applied (10 v/m)

Observations: No Susceptibilies

Inverter mode (UPS on battery) - RF applied (20 v/m)

Observations: 112 to $120 \mathrm{MHz}$ Overload LED on and Battery High LED flashing

@ $120 \mathrm{MHz}$ UPS output shuts down (pushed on/off buttom to restart)

120 to $150 \mathrm{MHz}$ Overload LED on and Battery High LED flashing

Testing performed by: Fred Gibson \& Roberto Jean-Pierre

D - 23 
Ambient and UPS Operating on AC

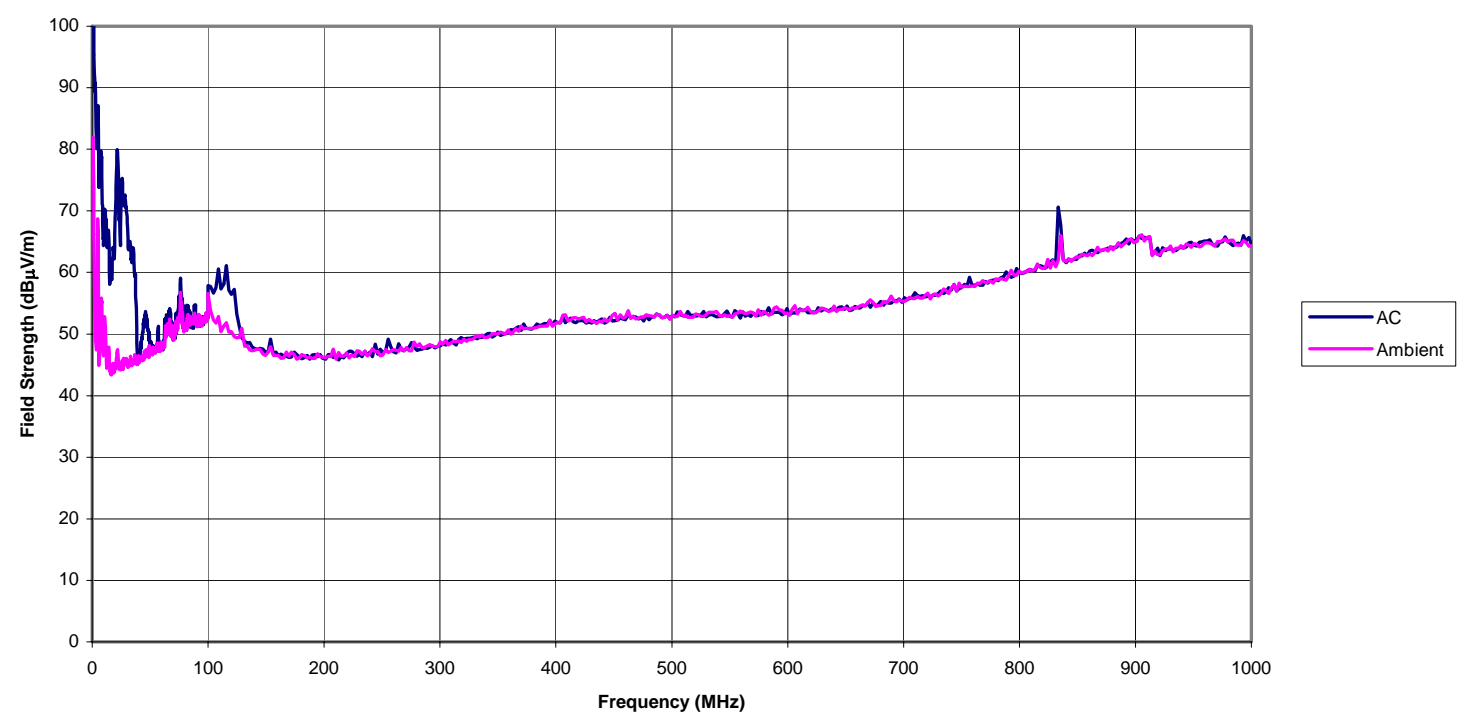

Ambient and UPS Operating on Battery

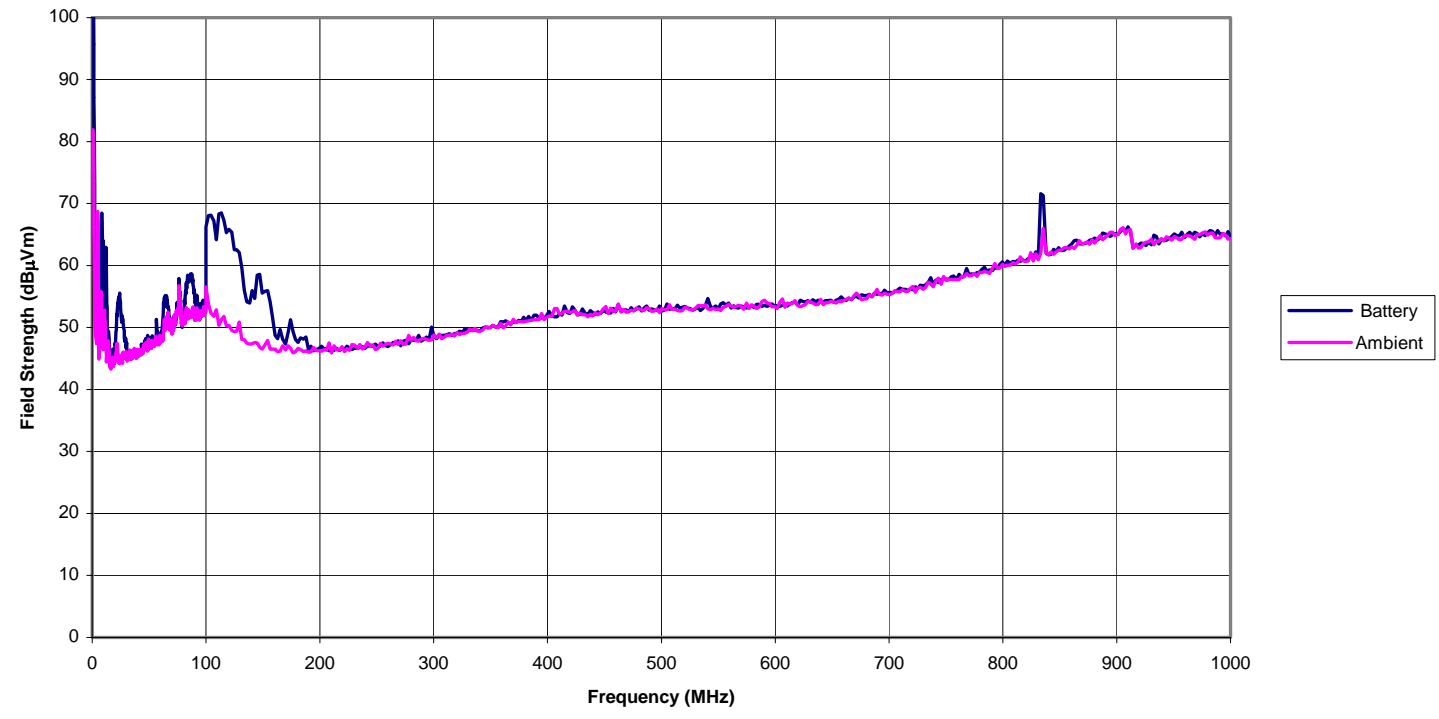


APPENDIX E

Equipment and Instruments used in Testing 


\section{Uninterruptible Power Supply (UPS)}

\section{Testing Protocol}

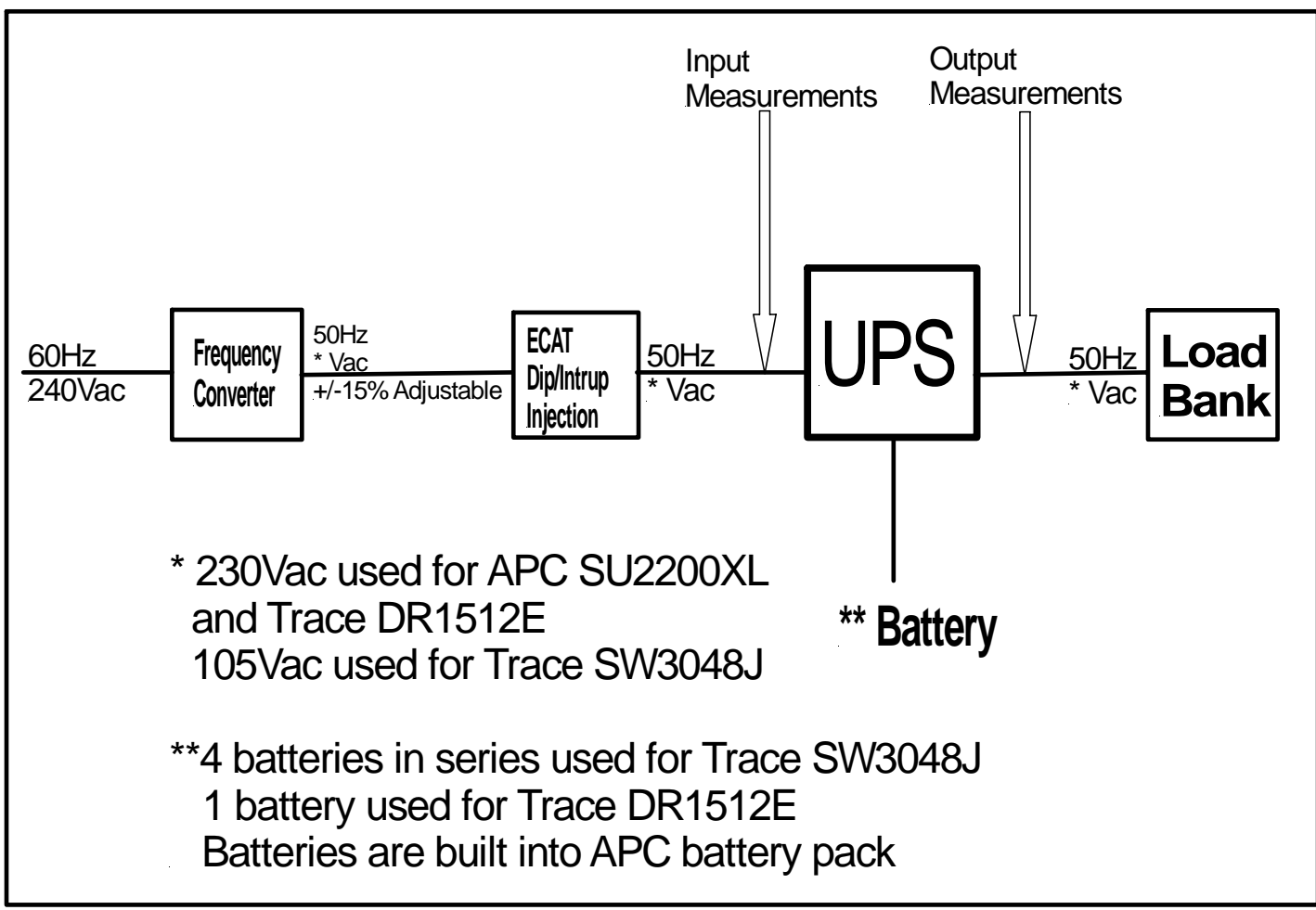

\section{Temperature}

Equipment used:

Russells Environmental Chamber

Visicomm Industries Frequency Converter 6.25KSS6050

Avtron K595 Loadbank

Instruments used:

Vaisala HMP235 Temperature/Humidity Meter

HP3456A DMM

AEMC 725 Harmonic Meter

Fluke 199C Scopemeter

Fluke 43B Power Quality Analyzer

Keytek ECAT E103 Controller

Keytek ECAT EP62 Dips/Interrupts

Keytek ECAT E4552 Coupler/Decoupler 


\section{Humidity}

Equipment used:

Russells Environmental Chamber

Visicomm Industries Frequency Converter 6.25KSS6050

Avtron K595 Loadbank

Instruments used:

Vaisala HMP235 Temperature/Humidity Meter

HP3456A DMM

AEMC 725 Harmonic Meter

Fluke 199C Scopemeter

Fluke 43B Power Quality Analyzer

Keytek ECAT E103 Controller

Keytek ECAT EP62 Dips/Interrupts

Keytek ECAT E4552 Coupler/Decoupler

\section{Condensation}

Equipment used:

Russells Environmental Chamber

Visicomm Industries Frequency Converter 6.25KSS6050

Avtron K595 Loadbank

\section{Instruments used:}

Vaisala HMP235 Temperature/Humidity Meter

HP3456A DMM

AEMC 725 Harmonic Meter

Fluke 199C Scopemeter

Fluke 43B Power Quality Analyzer

Keytek ECAT E103 Controller

Keytek ECAT EP62 Dips/Interrupts

Keytek ECAT E4552 Coupler/Decoupler

\section{Vibration/Shock}

Equipment used:

Vibration Test Systems DVC-4 Controller

Unholts Dickie 206 Shaker w/Amplifier

Visicomm Industries Frequency Converter 6.25KSS6050

Avtron K595 Loadbank

\section{Instruments used:}

HP3456A DMM

AEMC 725 Harmonic Meter

Fluke 43B Power Quality Analyzer

\section{Radiated emissions}

Equipment used:

Visicomm Industries Frequency Converter 6.25KSS6050

Avtron K595 Loadbank

Instruments used:

Agilent E4411B Spectrum Analyzer

ARA SAS-1/D Antenna 


\section{Electrostatic discharge}

Equipment used:

Visicomm Industries Frequency Converter 6.25KSS6050

Avtron K595 Loadbank

Instruments used:

Keytek MZ-15/ec ESD simulator

HP3456A DMM

AEMC 725 Harmonic Meter

Fluke 199C Scopemeter

Fluke 43B Power Quality Analyzer

Keytek ECAT E103 Controller

Keytek ECAT EP62 Dips/Interrupts

Keytek ECAT E4552 Coupler/Decoupler

\section{Conducted RF Disturbances}

Equipment used:

Visicomm Industries Frequency Converter 6.25KSS6050

Avtron K595 Loadbank

\section{Instruments used:}

HP 8648D Signal Generator

IFI SMX100 Amplifier

Agilent E4411B Spectrum Analyzer

HP3456A DMM

AEMC 725 Harmonic Meter

Fluke 43B Power Quality Analyzer

\section{Radiated RF field}

Equipment used:

EMCO GTEM model 5300

Visicomm Industries Frequency Converter 6.25KSS6050

Avtron K595 Loadbank

Instruments used:

EMCO 7110 interface unit w/RF probe

HP 8648D Signal Generator

IFI SMX100 Amplifier

HP3456A DMM

AEMC 725 Harmonic Meter

Fluke 43B Power Quality Analyzer 
Electrical fast transient (burst/100kHz ring wave)

Equipment used:

Visicomm Industries Frequency Converter 6.25KSS6050

Avtron K595 Loadbank

Instruments used:

HP3456A DMM

AEMC 725 Harmonic Meter

Fluke 43B Power Quality Analyzer

Keytek ECAT E103 Controller

Keytek ECAT E503 Ring wave module

Keytek ECAT E4552 Coupler/Decoupler

\section{Surge tests (combination wave)}

Equipment used:

Visicomm Industries Frequency Converter 6.25KSS6050

Avtron K595 Loadbank

Instruments used:

HP3456A DMM

AEMC 725 Harmonic Meter

Fluke 43B Power Quality Analyzer

Keytek ECAT E103 Controller

Keytek ECAT E501 Combination Module

Keytek ECAT E4552 Coupler/Decoupler 


\section{Power Supply}

\section{Testing Protocol}

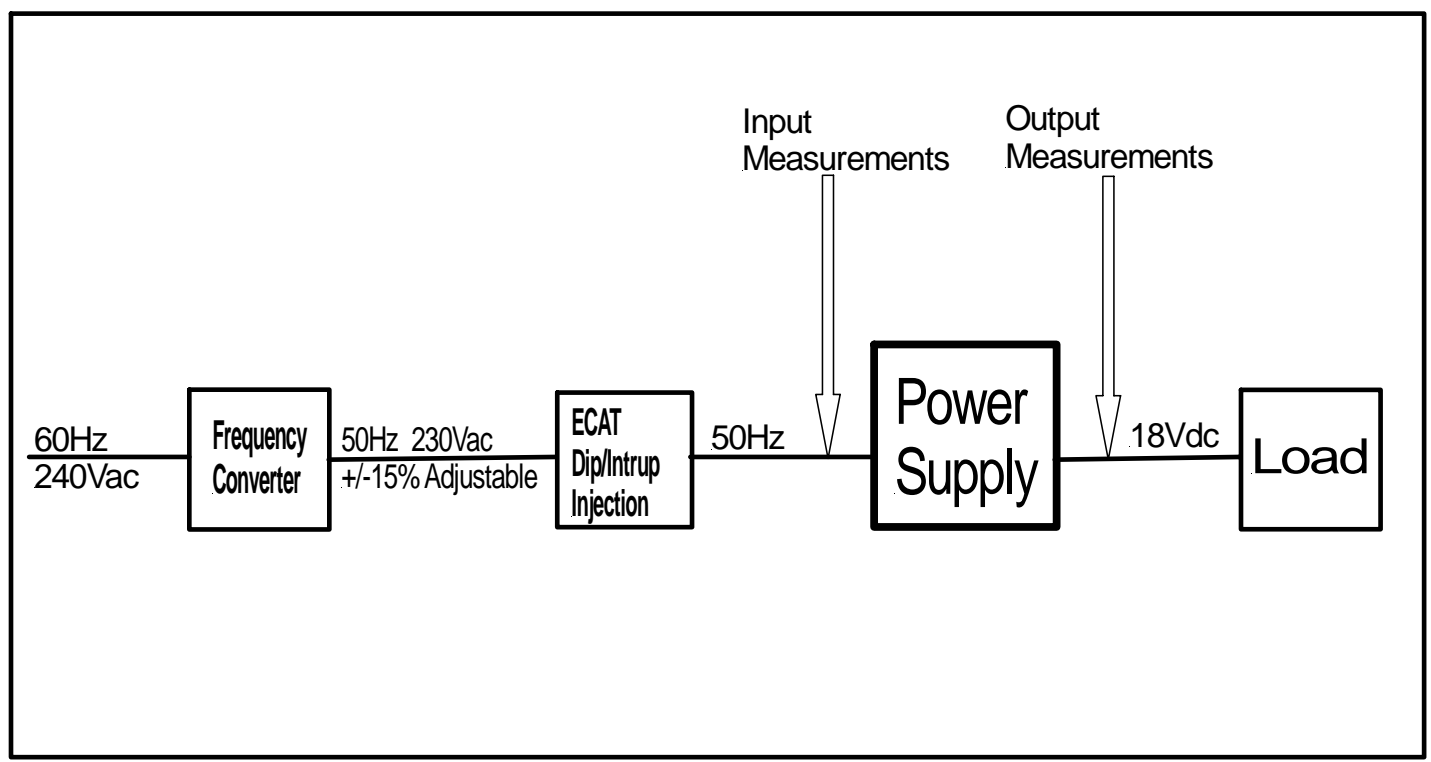

\section{Temperature}

Equipment used:

Russells Environmental Chamber

Visicomm Industries Frequency Converter 6.25KSS6050

12 ohms and 16 ohms loads

\section{Instruments used:}

Vaisala HMP235 Temperature/Humidity Meter HP3456A DMM

Fluke 199C Scopemeter

Fluke 43B Power Quality Analyzer

Keytek ECAT E103 Controller

Keytek ECAT EP62 Dips/Interrupts

Keytek ECAT E4552 Coupler/Decoupler 


\section{Humidity}

Equipment used:

Russells Environmental Chamber

Visicomm Industries Frequency Converter 6.25KSS6050

12 ohms and 16 ohms loads

\section{Instruments used:}

Vaisala HMP235 Temperature/Humidity Meter

HP3456A DMM

Fluke 199C Scopemeter

Fluke 43B Power Quality Analyzer

Keytek ECAT E103 Controller

Keytek ECAT EP62 Dips/Interrupts

Keytek ECAT E4552 Coupler/Decoupler

\section{Condensation}

Equipment used:

Russells Environmental Chamber

Visicomm Industries Frequency Converter 6.25KSS6050

12 ohms and 16 ohms loads

Instruments used:

Vaisala HMP235 Temperature/Humidity Meter

HP3456A DMM

Fluke 199C Scopemeter

Fluke 43B Power Quality Analyzer

Keytek ECAT E103 Controller

Keytek ECAT EP62 Dips/Interrupts

Keytek ECAT E4552 Coupler/Decoupler

\section{Vibration/Shock}

Equipment used:

Vibration Test Systems DVC-4 Controller

Unholts Dickie 206 Shaker w/Amplifier

Visicomm Industries Frequency Converter 6.25KSS6050

12 ohms and 16 ohms loads

Instruments used:

HP3456A DMM

\section{Radiated emissions}

Equipment used:

Visicomm Industries Frequency Converter 6.25KSS6050

12 ohms and 16 ohms loads

Instruments used:

Agilent E4411B Spectrum Analyzer

ARA SAS-1/D Antenna 


\section{Electrostatic discharge}

Equipment used:

Visicomm Industries Frequency Converter 6.25KSS6050

12 ohms and 16 ohms loads

\section{Instruments used:}

Keytek MZ-15/ec ESD simulator

HP3456A DMM

Fluke 199C Scopemeter

Fluke 43B Power Quality Analyzer

Keytek ECAT E103 Controller

Keytek ECAT EP62 Dips/Interrupts

Keytek ECAT E4552 Coupler/Decoupler

\section{Conducted RF Disturbances}

Equipment used:

Visicomm Industries Frequency Converter 6.25KSS6050

12 ohms and 16 ohms loads

Instruments used:

HP 8648D Signal Generator

IFI SMX100 Amplifier

Agilent E4411B Spectrum Analyzer

HP3456A DMM

Fluke 43B Power Quality Analyzer

\section{Radiated RF field}

Equipment used:

EMCO GTEM model 5300

Visicomm Industries Frequency Converter 6.25KSS6050

12 ohms and 16 ohms loads

Instruments used:

EMCO 7110 interface unit w/RF probe

HP 8648D Signal Generator

IFI SMX100 Amplifier

HP3456A DMM

Fluke 43B Power Quality Analyzer 
Electrical fast transient (burst/100kHz ring wave)

Equipment used:

Visicomm Industries Frequency Converter 6.25KSS6050

12 ohms and 16 ohms loads

Instruments used:

HP3456A DMM

Keytek ECAT E103 Controller

Keytek ECAT E503 Ring wave module

Keytek ECAT E4552 Coupler/Decoupler

\section{Surge tests (combination wave)}

Equipment used:

Visicomm Industries Frequency Converter 6.25KSS6050

12 ohms and 16 ohms loads

Instruments used:

HP3456A DMM

Keytek ECAT E103 Controller

Keytek ECAT E501 Combination Module

Keytek ECAT E4552 Coupler/Decoupler 


\section{Battery}

\section{Testing Protocol}

\section{Temperature}

Equipment used:

Russells Environmental Chamber (for 60 \& 110 Ah batteries)

Tenney Environmental Chamber (for 3.4 Ah battery)

Truecharge 10 battery charger (for $60 \& 110$ Ah batteries)

Nextgen Power Systems RR12350M battery charger (for 3.4 Ah battery)

25A, 0.48 ohms load bank (for $60 \& 110$ Ah batteries)

0.5A, 25 ohms load bank (for 3.4 Ah battery)

Instruments used:

Vaisala HMP235 Temperature/Humidity Meter

National Instruments SCXI data acquisition system

\section{Vibration/Shock}

Equipment used:

Vibration Test Systems DVC-4 Controller

MBIS SS500 power amplifier

MB Dynamics shaker

Nextgen Power Systems RR12350M battery charger

0.5A, 25 ohms load bank

Instruments used:

HP3456A DMM

National Instruments SCXI data acquisition system 
ORNL/TM-2004/26

\section{INTERNAL DISTRIBUTION}

1. Peter J. Chiaro, Jr.

2. Edward C. Fox

3. Fred R. Gibson

4. James E. Hardy

5. Kathy L. Johnson
6. J. Michael Whitaker

7. Central Research Library

8. ORNL Laboratory Records-RC

9-10. ORNL Laboratory Records-OSTI

\section{EXTERNAL DISTRIBUTION}

11. Massimo Aparo, International Atomic Energy Agency, Vienna, Austria

12. Michael Farnitano, International Safeguards Project Office, Brookhaven National Laboratory, Upton, NY 11973

13. Karl Ferstl, International Atomic Energy Agency, Vienna, Austria

14. Barbara Hoffheins, International Safeguards Project Office, Brookhaven National Laboratory, Upton, NY 11973

15. Susan Pepper, International Safeguards Project Office, Brookhaven National Laboratory, Upton, NY 11973

16. Mark Schanfein, International Atomic Energy Agency, Vienna, Austria 